\title{
Modelling of Coated Tilted Fiber Bragg Gratings
}

by

Nina Mamaeva, B.Sc.

A thesis submitted to the Faculty of Graduate and Postdoctoral Affairs in partial fulfillment of the requirements for the degree of

Master of Applied Science

in

Electrical and Computer Engineering

Ottawa-Carleton Institute for

Electrical and Computer Engineering

Carleton University

Department of Electronics

Ottawa, Ontario

(C) 2012, Nina Mamaeva 
Library and Archives

Canada

Published Heritage

Branch

395 Wellington Street

Ottawa ON K1A ON4

Canada
Bibliothèque et

Archives Canada

Direction du

Patrimoine de l'édition

395 , rue Wellington

Ottawa ON K1A ON4

Canada
Your file Votre référence

ISBN: 978-0-494-94263-5

Our file Notre référence

ISBN: $978-0-494-94263-5$
NOTICE:

The author has granted a nonexclusive license allowing Library and Archives Canada to reproduce, publish, archive, preserve, conserve, communicate to the public by telecommunication or on the Internet, loan, distrbute and sell theses worldwide, for commercial or noncommercial purposes, in microform, paper, electronic and/or any other formats.

The author retains copyright ownership and moral rights in this thesis. Neither the thesis nor substantial extracts from it may be printed or otherwise reproduced without the author's permission.
AVIS:

L'auteur a accordé une licence non exclusive permettant à la Bibliothèque et Archives Canada de reproduire, publier, archiver, sauvegarder, conserver, transmettre au public par télécommunication ou par l'Internet, prêter, distribuer et vendre des thèses partout dans le monde, à des fins commerciales ou autres, sur support microforme, papier, électronique et/ou autres formats.

L'auteur conserve la propriété du droit d'auteur et des droits moraux qui protege cette thèse. $\mathrm{Ni}$ la thèse ni des extraits substantiels de celle-ci ne doivent être imprimés ou autrement reproduits sans son autorisation.
In compliance with the Canadian Privacy Act some supporting forms may have been removed from this thesis.

While these forms may be included in the document page count, their removal does not represent any loss of content from the thesis.
Conformément à la loi canadienne sur la protection de la vie privée, quelques formulaires secondaires ont été enlevés de cette thèse.

Bien que ces formulaires aient inclus dans la pagination, il n'y aura aucun contenu manquant. 


\section{Abstract}

In recent years many research and development projects have been focusing on studying the fiber Bragg gratings. Fiber Bragg gratings have been used in sensors, lasers and communication systems. Some of the FBG based devices are already available, but a lot of questions are still to be answered. The researchers are working towards better understanding physical processes underlying operation of TFBGS (coated or bare). Better understanding of grating operation should facilitate new applications such as biosensing, chemical sensing and combination with other optical technologies and physical phenomena. The key requirements for commercialization of TFBGs and their wide application are going to be the low cost, compactness, and high volume manufacturability.

On the other hand the field of software development and programming techniques are also very popular. The behavior of electromagnetic light wave within a single mode fiber (SMF) will be analyzed using coupled mode theory (CMT). CMT is a suitable tool for obtaining quantitative information about the spectrum of a fiber Bragg grating. The goal of this project is to create a model for a SMF with a tilt angle and with a metal coating using commercial finite waveguide solver so that this model can be used in the future by other members of the research group. The procedure is carried out using FIMMWAVE (v 5.3.2) software developed by Photon Design and MatLab (2010b). Using a framework of optical waveguide theory, a firm understanding of the inner workings of TFBGs will be gained. The process of modeling a SMF will involve studying the process of mode coupling within a fiber, creating the list of modes using 
Fimmwave and finally acquiring the transmission spectrum using. This new model will then be compared to the published experimental results obtained by former members of the research group. 


\section{Acknowledgements}

I would like to thank my supervisor, Professor Jacques Albert, whose support, help, stimulating suggestions and encouragement helped me throughout this project.

I would also like to thank Professor Jacques Albert and Carleton University for their financial support during my period of studies.

I am very grateful to Tom Davies, chief optical engineer at Technix by CBS, for helping solve some problems with Fimmwave and MatLab throughout the research.

I would also like to thank my colleagues Albane Laronche, Lingyun Xiong, Aliaksandr Bialiayeu, Mohammad Zahirul Alam, Dr. Kseniya Yadav, Milad Dakka for help and cooperation, for interesting and useful discussions. I am obliged to Yanina Shevchenko for proofreading some of the chapters of this work and providing some valuable suggestions, also her friendship and support.

Finally I would like to thank all my friends for their support. Also I would like to express my deep gratitude to my family for their love and patience, to my father and my mother for inspiring my interest in natural sciences. 


\section{Table of Contents}

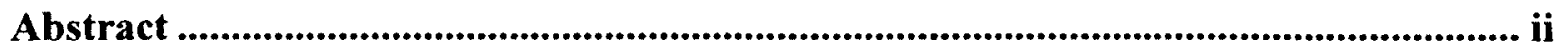

Acknowledgements ..............................................................................................................................iv

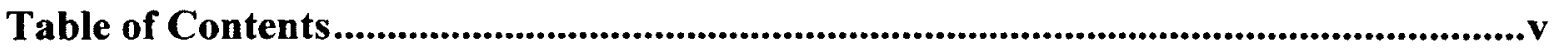

List of Tables ................................................................................................................................ vii

List of Figures ........................................................................................................................ viii

List of Appendices ...................................................................................................................................

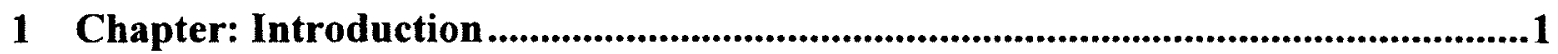

2 Chapter: Fiber Gratings. Fundamentals and Overview ...........................................5

$2.1 \quad$ Literature review...............................................................................................

$2.2 \quad$ Fiber Bragg grating operation principle ................................................................11

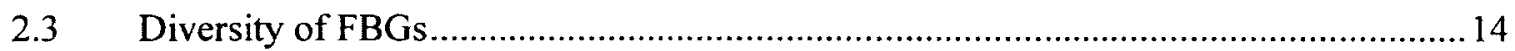

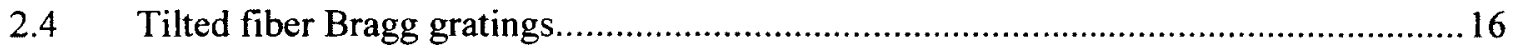

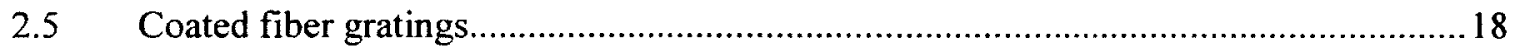

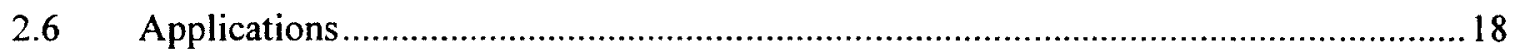

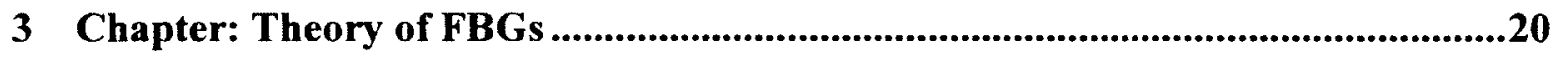

$3.1 \quad$ Coupled-wave analysis .....................................................................................21

3.2 Coupled-wave analysis for TFBGs........................................................................2

4 Chapter: Transmission characteristics of fibers.......................................................29

4.1 Classification and properties of modes in three-layer fibers ......................................29

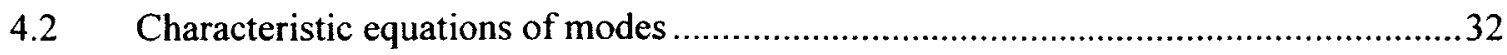

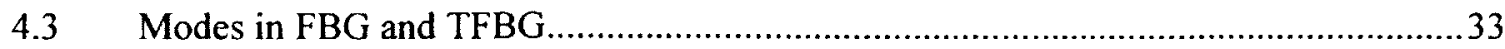

5 Chapter: Software and Simulation Technique ...........................................................36

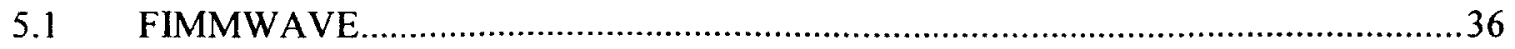


6 Chapter: Modeling of the Tilted Fiber Bragg Grating and Results 42

6.1 Building a Fiber Waveguide and Finding its modes..............................................42

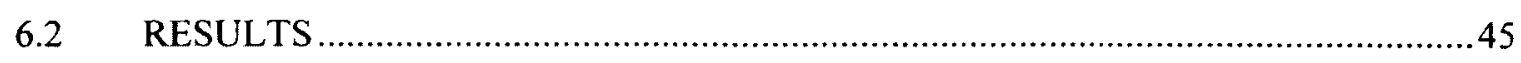

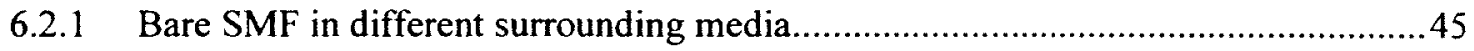

6.2.2 Gold-coated SMF in different surrounding media ..........................................48

6.2.3 Gold-coated TFBG in different surrounding media .......................................50

6.2.4 Spectral response of bare TFBG immersed in different surrounding media ...........52

6.2.5 Spectral response of Gold-coated TFBG immersed in different surrounding media59

7 Chapter: Conclusions and Future Work.....................................................62

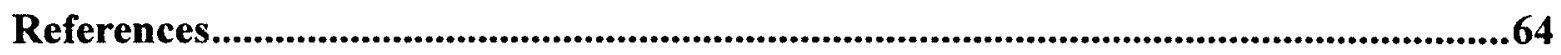

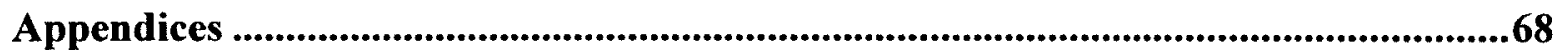

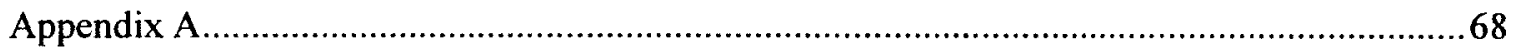

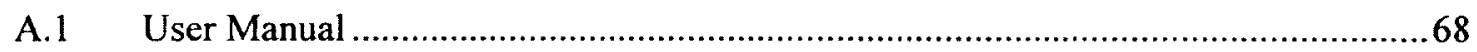

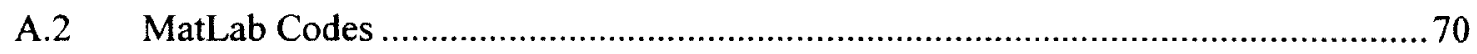




\section{List of Tables}

Table 6.1: Layers of a bare fiber and the properties of each layer .............................42

Table 6.2: Modes with various $m$ - and $p$ - vlues ...................................................44 


\section{List of Figures}

Figure 2.1: Uniform fiber Bragg grating operation principle. a). power spectrum of incident light, b). power spectrum for a transmitted wave, c). general wave propagation in FBG and d). power spectrum for a reflected wave.

Figure 2.2: Diagram of a step-index optical fiber showing an $x$-tilted fiber Bragg grating and some parameter definitions.

Figure 2.3: Typical Tilted fiber Bragg grating transmission spectrum.

Figure 3.1: Slab waveguide grating structure.

Figure 3.2: Example of reflectance and transmittance of grating reflectors. (Grating length $1 \mathrm{~cm}$, radius of the core of the fiber $1.8 \mu \mathrm{m}, n_{\text {core }}=1.47, n_{\text {clad }}=1.457, \Delta n=0.0003$ )

Figure 3.3: Diagram of the parameters associated with a TFBG. a. is the $x$ - tilted and b. is the $y$ - tilted grating.

Figure 4.1: Schematic drawing of a cross section of a three layer fiber with various refractive indexes and different radii of layers.

Figure 4.2: Dispersion of a fiber and electric field lines of some modes. [54]

Figure 4.3: Typical mode patterns observed: (a) ring, (b) and (c) bow tie, (d) and (e) quad

tie.

Figure 4.4: Three functions inside the integral for the coupling coefficient between the core mode $\left(\mathrm{HE}_{11}\right)$ and for a typical high order mode $\left(\mathrm{HE}_{\mathrm{mn}}\right)$, depending on the polarization of the input mode. The top row corresponds to S-polarized input light, and the bottom row to P-polarized light [39]. 
Figure 5.1: The intensity of even and odd components of one of the cladding modes of a fiber. Top row 2-D and bottom row in 3-D. Intensity is measured in $\mathrm{nJ} / \mathrm{m}^{3}$; the $x$ - and $y$ axes are in $\mu m$.

Figure 6.1: The cross section of chosen fiber (left) and its refractive index profile (right)

Figure 6.2: Polarization of found modes as function of resonance wavelength found for bare SMF-28 surrounded by air.

Figure 6.3: Polarization of found modes as a function of resonance wavelength found for bare SMF-28 immersed in water.

Figure 6.4: Imaginary part of modes propagating in Au-coated fiber as a function of wavelength; in air.

Figure 6.5: Imaginary part of modes propagating in Au-coated fiber as a function of wavelength; in water.

Figure 6.6: Transmission spectrum as a function of wavelength for the 10-degree grating surrounded by Air. .51

Figure 6.7: Transmission spectrum as a function of wavelength for the 10-degree grating surrounded by water. .52

Figure 6.8: Transmission spectra as a function of wavelength for the surrounding refractive index $n_{\text {out }}$ changing from 1 to 1.35 . .54

Figure 6.9: Same spectra as Fig. 6.8 but zoomed in. .55

Figure 6.10: Transmission loss of a certain resonance as a function of wavelength. .56 Figure 6.11: Effective index of a mode as a function of refractive index of surrounding media. 
Figure 6.12: a). Typical experimental TFBG transmission spectrum (SMF-28 fiber, $\theta=6^{\circ}$ ) measured in air. b). Several measurements with various refractive indices of the outer medium near the Bragg resonance. (c) Same spectra as (b) but zooming in on a particular resonance near $1535.5 \mathrm{~nm}$ [66].

Figure 6.13: Tramsission loss of a modes propagating in a gold coated SMF-28. 59

Figure 6.14: Effective index of a mode $\left(n_{e f f} \approx 1.3528\right)$ as a function of refractive index of surrounding media.

.60 


\section{List of Appendices}

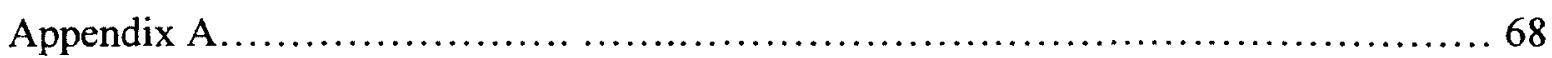

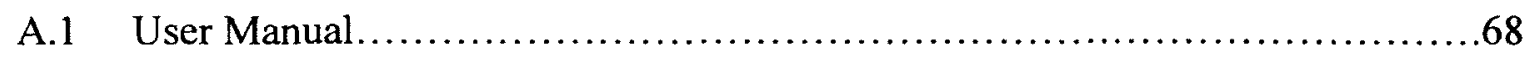

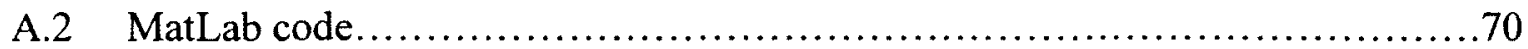




\section{Chapter: Introduction}

Fiber optics is currently a very progressive and popular area of research. The demand for higher speed, more accurate devices and computing performance is growing rapidly, so the researchers are trying to find solutions to fulfill the demand, and photonics is a potential research area of the future devices. As a result, intense interest has focused on fiber Bragg gratings because of their ability to be used in many different applications such as rare-earth doped fiber lasers [1], wavelength division multiplexing [2], mode couplers [3], hybrid fiber/semiconductor lasers [4], grating based sensors [5] and many more. The other potential fiber Bragg grating applications are still in development. The main areas where FBGs are one of the main component is the telecommunication systems $[6,7]$ and sensing systems $[8]$.

In order to understand the process of light transmission through an optical fiber, the light transmission speed and the field distributions in cross-section of the fiber should be investigated. Thus the fiber modes need to be solved. There are several types of fibres hence different fiber modes will be supported differently. The fibers can be classified as single-mode fiber (SMF) and multimode fibers (MMF). Single-mode fiber can only support one core mode, whereas multimode fibers can support many core modes. As well as core mode, fibers can also support cladding modes, leaky modes and radiation modes, depending on the core, cladding and surrounding medium. The other classifications of fibers are weakly or strongly guided step-index or graded-index fiber. Different fibers have their significance in different applications. 
A lot of optical devices use the principle of mode couplings and most of them are fiber Bragg grating based devices. The fiber Bragg gratings (FBG) were discovered by Hill et al. [9]. There are several writing techniques that have been developed for FBG writing. One of the first ones are the ultra-violet (UV) writing technique [10] and the phase mask technique [11]. The FBG based devices are compact in geometry, cost efficient, have low insertion loss and are immune to electromagnetic interference. Those are just some of the advantages of FBG based devices compared to the bulk devices. These are the reasons why FBGs play a very important role in the fiber optic communications and sensor systems.

A tilted fiber Bragg grating (TFBG) is a fiber Bragg grating with the prating plane inclined at a small angle relative to the $x$ or $y$-axis. In the TFBG, the modes are coupled between the forward propagating core mode to backward propagating core mode (Bragg), and forward propagating core mode to backward propagating cladding modes. Therefore both a core mode resonance and numbers of cladding mode resonances appear simultaneously [12]. Using the core mode back reflection as a reference wavelength in $\mathrm{SMF}$, it is possible to measure the perturbations such as surrounding refractive index using the cladding mode resonance shift. The sensitivity of TFBG to the surrounding media can be extended to a next level of sensitivity. The TFBG can be coated with a metal layer, and this coating will act as a transducer between the surrounding media and the fiber and as a result, the response will be different.

Suitable software and programming language are very important in a simulation. There are several factors, such as code reusability, speed and compatibility, should be taken into account when choosing the programming language. A computer simulation is 
quite significant in optical fiber research field. The use of expensive and delicate systems and equipment can be omitted until the design is optimized. Most of the times environmental and noise factors play a crucial role during an experiment, they may change the results dramatically. The theoretical results can be obtained using the simulation and then the theoretical and experimental results can be compared and the factors that affect the system can be found.

This thesis is organized as follows. The second chapter will contain some background of the FBGs, different types and the operation principles will also be explained. The TFBGs and coated TFBGs will also be described as well as some of the research that has been done on this topic. Finally, some of the applications where FBGs can be used will be listed. Chapter 3 will describe the theory behind FBGs that has been used for the simulation. Coupling coefficient and the reflectance and transmittance between core and cladding modes in FBGs as well as in TFBGs will be derived. Some examples of transmission and reflection spectra will be presented. The fourth chapter will be focused on the mode couplings within a step-index fiber. Different types of modes are examined and used to explain the field propagation in FBGs and TFBGs. The software and the programming language and techniques will be described in Chapter 5. It will also demonstrate how these techniques can be applied to solve the fiber Bragg grating problem. The advantages and disadvantages of the programming languages will be discussed. The application of the simulation, the full description of a model will be presented in Chapter 6. Some results that were obtained for bare fibers and coated fibers will be presented. Then the transmission spectra that were obtained using the simulation will be plotted and then all results will be compared to the experimental results acquired 
from other members of the research group. Final conclusions and recommendations for future work will be presented in Chapter 7 . 


\section{Chapter: Fiber Gratings. Fundamentals and Overview}

\subsection{Literature review}

First fiber Bragg gratings were introduced in 1978 by K.O. Hill et al at the Canadian Communications Research Centre (CRC), Ottawa, Ont., Canada [9]. They launched a beam of an intense UV-light through the Ge-doped core optical fiber and it was noticed that the intensity of the reflected light started to increase until eventually almost all light was reflected from the end/tip of the fiber. After spectral analysis was performed it was determined that interaction of UV light with Ge-doped silica resulted in formation of a periodic filter (subsequently called Hill gratings) [9]. Two light waves propagating in opposite directions created an interferometric pattern in the fiber core, which lead to a permanent periodic perturbation of the refractive index in it. This phenomenon was possible due to photosensitivity effect, which is very common for the silica materials with various dopants.

While Hill's approach was based on launching UV-Light in the fiber's core; in 1989 Meltz et. al. introduced a new technology for fabricating Bragg gratings. A grating was formed by exposing a short length of a bare optical fiber through the side to a pair of intersecting UV beams [10]. They demonstrated reflection gratings operating in the visible part of the spectrum (571-600 nm) using their new holographic technique. This scheme provided a possibility to shift the Bragg condition to increase the wavelength diapason $(1200 \mathrm{~nm}-1500 \mathrm{~nm})$ by varying an angle between the interfering beams [13]. Since then, a lot of new ideas and methods for writing the grating were proposed, which may look similar, but differ radically on the microscopic scale. This field remains a very 
active area of research, which leads to development of modern optical communications and sensor systems.

A lot of fiber grating related articles have appeared in the scientific literature and conferences. A general literature review was conducted to understand the fundamentals of FBGs and TFBGs, including theory and simulation techniques. One of the most important functions of the fiber grating is its ability to couple a guided mode to radiation modes in the fiber. In order to understand the light transmission in fiber, the field distribution in the in the fiber needs to be known, thus the modes need to be solved. To find the effective index of a mode and its field distribution several methods can be used such as methods that use matrix to express the fields. Some of the commercial optical fiber solvers can be used to find the field distribution in a fiber.

The principle of mode couplings is being employed in lots of optical devices, such as optical couplers, optical mode converters etc. The theory for mode couplings in optical waveguides was developed [14] even before the FBG was invented. There are several ways to analyze the mode coupling in fibers; coupled mode theory (CMT) is one of the most popular and the most developed $[15,16]$. Erdogan et al. did the most detailed work for calculating the coupling constant between modes [16]. He solves analytically the coupling constants for a three-layer step index fiber grating. The theory proposed accurately models the transmission in gratings, which support both counterpropagating (short-period) and co-propagating (long-period) interactions.

Coupled mode theory (CMT) approach features clear physical concept and effective method for analyzing interactions between different modes in optical fiber gratings. Because the index difference at the waveguide boundary is considered, the CMT 
is the more rigorous approach and is convenient for simulating the spectrum. In 1996 a spectral analysis of tilted fiber Bragg gratings was carried out by Erdogan and Sipe [15] on radiation-mode coupling with the complete CMT equations when the tilt angle varied from 0 to 15 degrees. Good agreement was obtained between the theoretical and the experimental results.

In this approach, one calculates the grating-induced coupling coefficients between the guided mode and a whole set of radiation modes; these coefficients are then summed up to obtain the scattering loss, and the scattered field can be determined by combining all the radiation modes. Because the index difference at the waveguide boundary is naturally taken into account, this is one of the more rigorous approaches.

In 2000 Lee and Erdogan analyzed in greater details the interaction between core mode and hybrid cladding modes and between core mode and higher-order core modes in reflective and transmissive tilted fiber gratings. In their paper it was shown that in the transmissive tilted grating a strong coupling occurs between core mode and cladding mode for almost any tilt angle, except angles close to $90^{\circ}$. And in a reflective grating, strong coupling occurs between core mode and the cladding modes occurs only for angles less than $5^{0}$, whereas coupling to higher-order modes occurs at angles greater than $5^{0}$. The numerical simulation was carried out using CMT. [17]

In $2009 \mathrm{Lu}$ et al. proposed simplified CMT approach to perform analysis of for radiation-mode coupling in TFBG. In their work, they consider the coupling between the core mode and the continuum of radiation modes, based on consideration of the vectorial phase-matching conditions and the phase terms of the complete CMT equations. They demonstrated similar results as Erdogan did in 1996 [15]. Lu et al. presented a detailed 
analysis on the relationship between radiation-mode loss and tilt angle ranging from 1 to 45 degrees for two orthogonal polarization states. With this model, they derived the same analytical formula for nonparaxial scattering as from the VCM (volume current method) analysis. The simulation showed that the radiation-mode coupling possesses a polarization dependence property, and particularly when the tilt angle reaches 45 degrees, the two polarization states can be highly separated. They also investigated the properties of $45^{0}$-tilted grating, which provided effective design guidance for achievement of highperformance in-fiber polarizer and polarization splitters [18].

In 1996 Vengsarkar et al. [19] introduced a long -period grating (LPG) technology that can be used as in-fiber, low-loss, band-rejection filters. In their work they described the interaction between the guided fundamental mode in a SMF and forward-propagating cladding modes in long period gratings. They developed a theory, based on CMT, for these filters and performed some experiments, which showed that all-fiber filters are versatile devices with low insertion losses and low back-reflections and have excellent polarization insensitivity.

In 2003 Anemogiannis et al. [20] presented a numerical method, which can simulate non-tilted fiber gratings. He calculated the transmittance of long-period (LP) grating, which has arbitrary azimuthal/radial refractive index variations. The interactions between core mode and high-azimuthal-order cladding modes were taken into account. The method was based on the CMT oh hybrid modes in step-index optical fibers and the transfer-matrix method was used for generation of the mode radial fields. As a result, the transmission spectra were built and the resonance features in it were explained by the coupling between the modes. Even though this particular numerical method was built 
only for the LPFG, it can also be used for simulation of fiber Bragg gratings with modified CMT equations.

General properties, most common fabrication techniques and the most important areas of application one can find in [21]. Vasiliev et al. presents the basic theoretical equations describing spectral properties of the LPG and the comparison to the spectrum obtained experimentally.

In 2001 Lee and Erdogan analyzed mode couplings in tilted fiber gratings [17]. They determined that a number of modes can be formed through the mode conversion process in the gratings and with linear combination of four different modes. Properties of both the single-sided and double-sided tilted grating for core-cladding mode coupling were analyzed in detail. The transmission spectra built using the numerical model predicted by the coupled-mode theory agreed with the transmission spectra build experimentally.

In the same year Li et al. [22] introduced another analytic approach to calculate the radiation pattern of TFBG using volume current method (VCM). Theoretical results are derived and discussed as well as compared to experimental measurements. The results of their analysis showed that tilted fiber gratings have the ability to act as fiber taps and efficiently couple light out in a highly directional fashion. The theory also showed that the greatest polarization selectively occurs for radiation coupled out at $90^{\circ}$ with respect to the fiber axis, and this can be achieved by a grating with a $45^{\circ}$-tilt angle.

Number of useful devices is employing the polarization-sensitive mode-coupling characteristics of TFBG. A thorough and extensive theoretical and numerical analysis of TFBG was presented by Walker et al. [23] using VCM. They review the limitations and 
shortcomings of this formulation as well as further clarify the physical relationships between grating's structure and its radiation field characteristics.

In $2006 \mathrm{Li}$ and Brown developed a waveguide scattering analysis based on the CMT and sets of hybrid HE and EH guided modes in a tilted fiber grating [24]. With this approach they were able to get some analytical results for nonparaxial scattering as from the VCM analysis. Their numerical simulation showed that VCM provides a good estimate of the scattering profile, except at very small scatter angles. In conclusion they stated that there are minor differences between CMT and VCM except at very small scatter angles.

In $2006 \mathrm{He}$ et al. [25] presented a new type of optical sensor based on a thin metallic film and long-period fiber gratings for measuring small changed in refractive index of analyte. CMT was used for theoretical analysis of the structure. The variation of the surrounding media was determined by looking at the change of the transmitted core mode power, which was calculated using two-mode coupled-mode equations at a fixed wavelength. The numerical simulation results showed that this configuration could be used as highly sensitive amplitude sensor.

Further, in $2009 \mathrm{Lu}$ et al. investigated the influence of the mode loss on the refractive index sensors made out of coated fiber Bragg grating. They demonstrated through a simulation that the gating length must be smaller or comparable with the propagation length of "surface plasmon polariton - mode" in order to achieve effective coupling. In other words, in order to achieve effective mode coupling with the help of waveguide grating, the grating length is bounded by the shortest propagation length of the modes in lossy waveguides [26]. 
In $2010 \mathrm{Lu}$ et al. [27] investigated theoretically the polarization effects in tilted fiber Bragg grating (TFBG) refractometers. The polarization effects may have a very big influence on the sensor performance, thus should be considered to achieve an accurate measurement of surrounding refractive index. He also discusses the ways to reduce reduction of the polarization effects, such as all of the components between the optical source and the TFBG should be purely polarization independent or polarization maintained with respect to the TFBG grating plane, though this criteria is very difficult to achieve. One way to achieve this is to use the linear polarizer or polarization controller and the other is to average the results for orthogonal polarization states. This is needed for the experimental results, in theory, for simplicity only a certain polarization can be taken into account.

Most recently Thomas et al. [28] presented a complete vectorial analysis of cladding mode coupling in highly localized fiber Bragg grating. They show how the reflected cladding modes can be analyzed taking into account their vectorial nature, orientation and degeneracies. The intensity and polarization distributions of the observed modes are related to the dispersive properties, as well as show rapid transitions, strongly correlated with changes in the coupling strength.

\subsection{Fiber Bragg grating operation principle}

Fiber Bragg grating (FBG) is a periodic structure that can be written, for most of the cases, in the core of a fiber. It reflects a narrowband portion of incident light and transmits the rest. The wavelength of reflected band depends on the periodicity of the grating. 
FBGs can be manufactured by following techniques that can be divided into two categories: interference and photomasking [13]. During the photomasking process a mask is placed between the UV light source and the photosensitive fiber. The shadow of the mask then gives the grating structure depending on the intensity of the incident light. As mentioned above, the principle of the interference technique is in periodic altering of the refractive index by UV - light illumination. The exposure creates the periodic perturbation of permanent refractive index, $\delta n$, in core of the fiber. Refractive index change $\delta n$ is positive for high germanium doped fibers with a magnitude ranging from $10^{-5}$ to $10^{-3}[29]$.

Refracted index modulation can be represented by [30]:

$$
n(z)=n_{c}+\delta n \cos \left(\frac{2 \pi}{\Lambda} z\right)
$$

where $n_{c}$ is the refractive index of the core, $\delta n$ is an amplitude of the core index change, $z$ is a fiber axial direction and $\Lambda$ is the grating period.

FBG alters propagation of light in the fiber's core, depending on the grating's type it can backscatter light or deflect it into the cladding in at a certain angle. Scattering of light in a straight Bragg grating can be explained using ray optics. Fiber grating is similar to a multilayer dielectric mirror to a certain extent, but instead of having small amount of layers with high refractive index, fiber Bragg grating has thousands of layers with small refractive index modulation. A typical layout of a uniform fiber Bragg grating with input and output signal is shown on Figure 2.1 [31]: 
a.

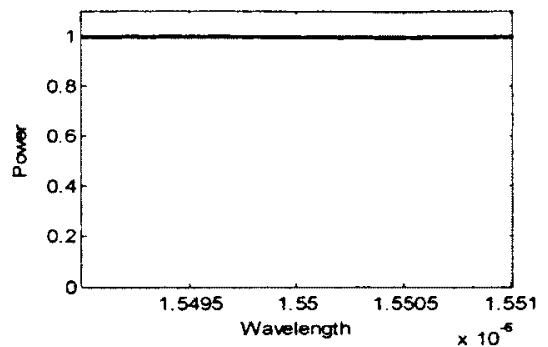

b.

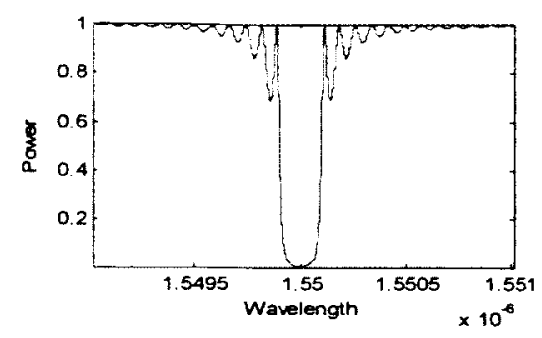

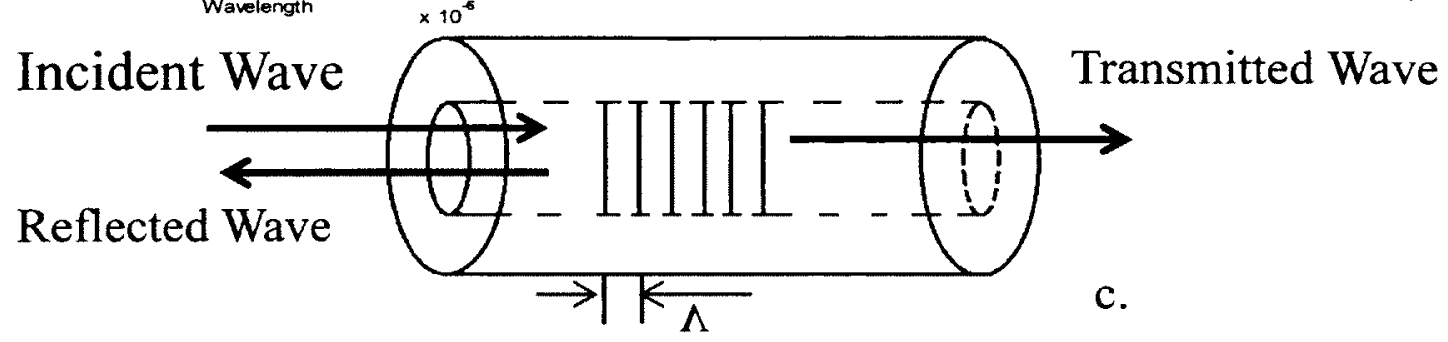

d.

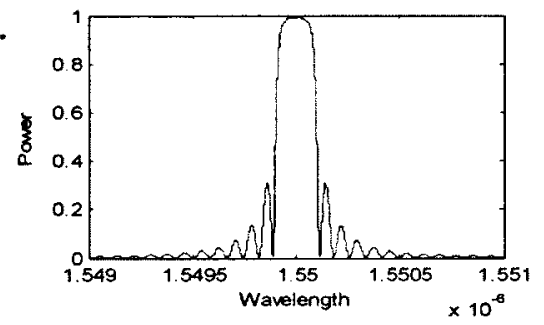

Figure 2.1: Uniform fiber Bragg grating operation principle. a). power spectrum of incident light, b).

power spectrum for a transmitted wave, c). general wave propagation in FBG and d). power spectrum for a reflected wave.

The incident light while propagating through a grating is being reflected by a small amount at each periodic refractive index change. All the reflected waves ("portions") are then combined at a particular wavelength and the strongest mode couplings occur, if each of these reflections are in phase. This is called a phase matching condition. Bragg condition occurs only if the momentum and energy conservation are satisfied for one particular wavelength. This requirement means that the sum of the incoming light wave vector $k_{i}$ and grating vector $k_{g}$ should equal to the scattered wave vector $k_{r}$ [31].

$$
k_{i}+k_{g}=k_{r}
$$


In the single mode waveguide the wavelength at which the momentum conservation

occurs, is called a Bragg wavelength $\lambda_{\text {Bragg }}$ [4]. The wave vector of an incident wave is defined as:

$$
k_{i}=\frac{2 \pi n_{e f f}}{\lambda_{B}}
$$

Since there is only one mode in a single mode fiber, reflected wave will have the same vector as the incoming wave, but opposite in direction. Assuming that the grating wave vector is:

$$
k_{g}=2 \pi / \Lambda
$$

where $\Lambda$ is the grating period. Then equation 2.2 can be written as:

$$
\frac{2 \pi n_{e f f}}{\lambda_{B}}+\frac{2 \pi n_{e f f}}{\lambda_{B}}=\frac{2 \pi}{\Lambda}
$$

or

$$
\lambda_{B}=2 n_{e f f} \Lambda
$$

where $n_{\text {eff }}$ is the effective refractive index.

Therefore, the grating acts as a filter, which reflects the light with the wavelengths close to Bragg wavelength and transmits the rest.

\subsection{Diversity of FBGs}

Since the moment fiber Bragg gratings were discovered, considerable research has been done in this field and several more types of fiber gratings were invented.

The diversity of grating types can be explained by the research in fabrication of fiber gratings. There are several distinct types of fiber Bragg grating structures: long- 
period (LPG) and short-period (SPG) Bragg gratings, tilted Bragg gratings (TFBG), chirped gratings, phase -shifted gratings and a combination of grating designs [31]. LPGs and SPGs have been analyzed theoretically and experimentally by Erdogan in 1997 [16]. $\mathrm{He}$ modeled and measured the transmission in gratings that support both counterpropagating (short-period) and co-propagating (long-period) interactions. Specifically tilted SPG have been analyzed experimentally by Laffond and Ferdinand in 2001 [32]. In this work they investigated the changes in the transmission spectrum of long period fibre gratings and tilted short-period fibre Bragg gratings versus the refractive index of the surrounding medium. There are several structures of FBGs, most common are uniform with positive-only index change, Gaussian-apodized, raised-cosine-apodized with zero-dc index change, chirped, discrete phase shift (of $\pi$ ), and superstructure [33].

Different grating types can be used in different applications depending on their properties. Some of applications require a nonuniform grating to reduce the unwanted side-lobes that appear in uniform grating spectra. There are many other reasons to adjust the optical properties of a fiber grating by tailoring the grating parameters along the fiber axis. It has been known that apodizing the coupling strength of a waveguide grating can improve the side-lobe suppression and can produce a reflection spectrum that more closely approximates the desired shape while maintaining narrow bandwidth [34].

Moreover, the grating can be modified to add other characteristics, such as chirp, which is a linear variation in the grating period. Chirped fiber gratings are useful for dispersion and polarization compensation, controlling and shaping short pulses in fiber lasers $[35,36]$. 


\subsection{Tilted fiber Bragg gratings}

Meltz et. al. were first to introduce the tilted fiber gratings in 1990 [37]. Tilted grating (Fig. 2.2) is a fiber grating with planes of the grating being rotated at a certain angle relatively to the light propagating in the core. Laffont and Ferdinand were monitoring the envelope of the resonances produced by a tilted grating as a function of the surrounding refractive index (SRI) [32]. As the SRI increased, high order cladding modes became leaky modes and as a result, the area covered by the envelope of the resonance distances decreased.
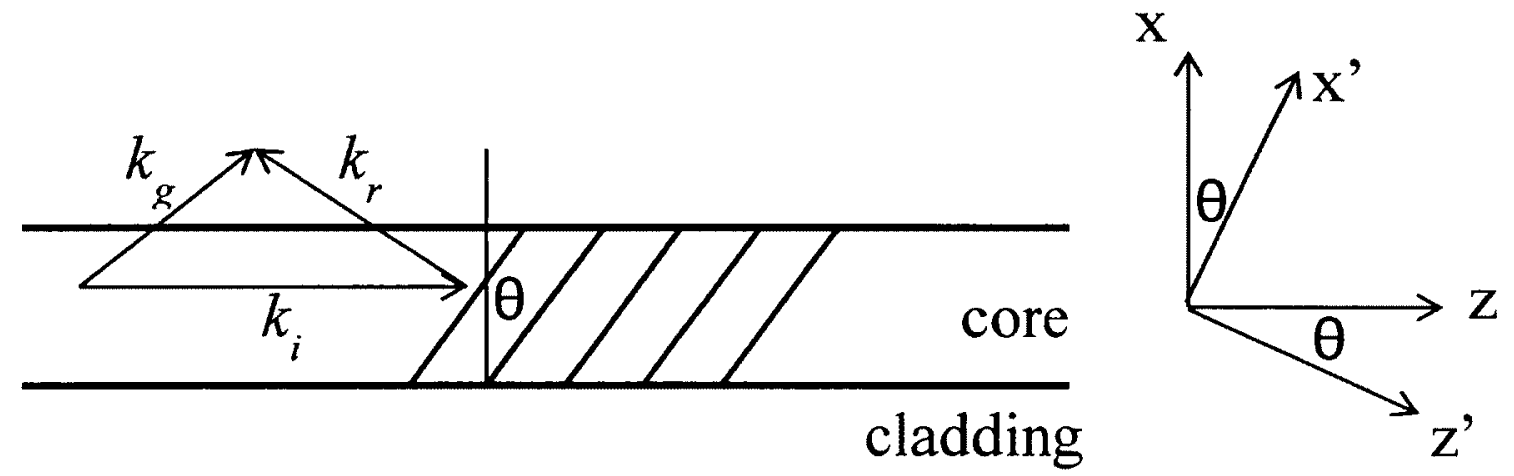

Figure 2.2: Diagram of a step-index optical fiber showing an $x$-tilted fiber Bragg grating and some parameter definitions.

It was discovered that both a core mode resonance and several cladding mode resonances appear simultaneously (Figure 2.3 ). The advantage of TFBG spectrum is that all the cladding mode resonances occupy a range of spectrum from a few tens up to about 200 nanometers The cladding mode resonances are sensitive to the external environment (refractive index, deposited layer thicknesses, etc.) and to physical changes in the whole fiber cross-section (for instance, shear strains arising from bending for instance), while the core mode (Bragg) resonance is only sensitive to axial strain and temperature [38]. 
Phase matching condition (Equation 2.2) predicts that at any wavelength shorter than Bragg wavelength $\lambda_{B}$ can be coupled to cladding modes by any grating. Though experimental results contradict this statement [39]. Previous experiments [15, 40] demonstrated that such coupling is much stronger for TFBGs than for FBG. This is due to the Bragg diffraction formation: light from the core mode hits each grating plane of the FBG at right angle and is reflected backwards; thought when the grating planes are tilted, light is reflected off axis and each grating plane reflects a small portion of light towards the cladding. This increases the growth of the backward propagating cladding mode at phase-matched wavelengths. The cladding modes that will have the strongest coupling are then determined by the tilt angle.

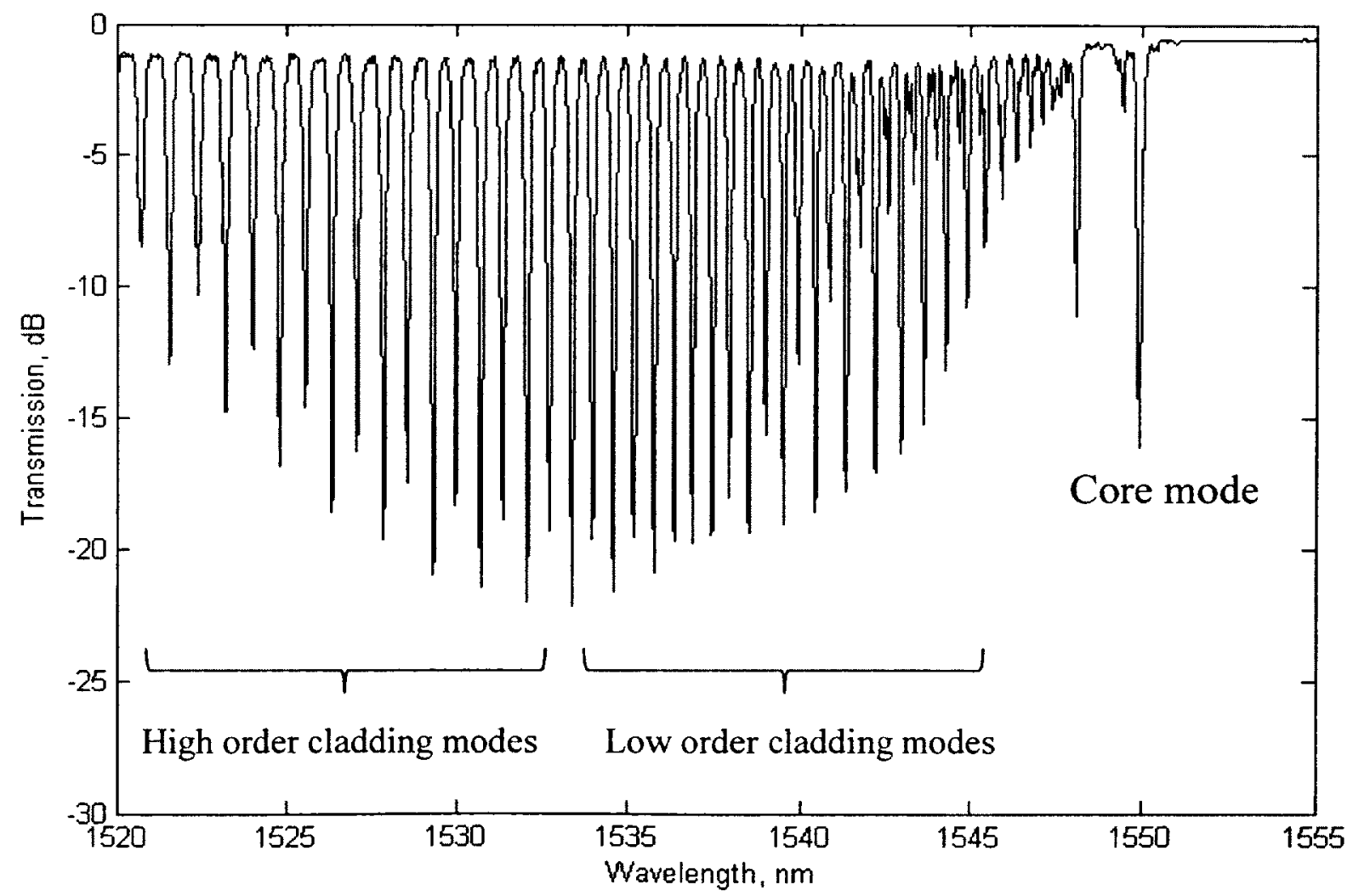

Figure 2.3: Typical Tilted fiber Bragg grating transmission spectrum. 


\subsection{Coated fiber gratings}

As it was mentioned above, TFBGs are very sensitive to the refractive index of the surrounding media. This property can be used to sense other parameters as well by coating fibers with materials that react to different modulations such as chemical or physical. These coatings act as transducers between the surrounding media and fiber, and as a result of deposition of a coating the spectrum will be different. The response of this structure depends on the overlap between the guided waves and the coating, as well as the refractive index of the coating, its thickness and absorption, and the refractive index of the medium surrounding the coating. One of the main applications of the coated TFBGs is in chemical sensing and refractometry.

\subsection{Applications}

The fiber Bragg gratings written by UV light into the core of an optical fiber have developed into an important component for many applications in fiber-optic communication and sensor systems. This technology enabled the fabrication of a variety of different Bragg grating devices that were not possible to build before. A good example of such a device is FBG dispersion compensator [41]. Overall, the research has been focusing mostly on the development of the FBG-based devices for use in fiber optic communications or fiber optic sensor systems, as well as in laser systems and less so on other non-linear applications.

In recent years fiber optic telecommunication systems, used for fast, efficient and low-cost data transfer and storage, became a very popular area of research. Fiber Bragg 
gratings became one of the most important components in telecommunication applications. FBGs has been used in wavelength converters [42], Raman amplifiers [43], add/drop multiplexers [44], phase conjugators [45], temperature, pressure, strain sensors $[46,47]$, semiconductor lasers $[48]$ etc.

The progress in the material science made possible the doping of the fiber core with different ions in order to decrease propagation loss and increase the efficiency of fiber lasers. Fiber Bragg gratings must withstand exceptionally high temperatures and high optical field resistance during high power fiber laser operation. The combination of high spectral selectivity and low resonator insertion loss of fiber Bragg gratings has enabled a variety of devices that are not possible with electrical strain gages [49].

Optical sensing systems is one of the most promising areas of research, where fiber Bragg gratings play a very important role. As it was described earlier, the parameters and the responses of the fiber gratings are very sensitive to the surrounding environment, such as temperature, strain, refractive index, vibration and pressure. Thus, FBGs as well as TFBGs can be used in development of physical sensors, refractive index sensors and biochemical sensors, which can be used in different industries such as biomedicine, oil exploration, structural health monitoring and many more. 


\section{Chapter: Theory of FBGs}

A lot of methods have been developed for the analysis of the field propagation in gratings and interaction with media surrounding the fiber. The most common technique that describes the behavior of EM fields within fiber gratings is CMT [50]. It is relatively simple and very accurate in modeling the optical properties of fiber Bragg gratings.

Coupled mode theory (CMT) was first developed in the early 1970's before fiber Bragg gratings were discovered. Yariv and Snyder were some of the pioneers who introduced CMT to guided-wave optics to understand the process of the mode coupling in optical waveguides $[50,51]$. The theory was initially developed for the uniform gratings, however, Kogelnik [52] extended the model to cover aperiodic structures.

CMT focuses on counter-propagating fields inside the grating structure, obtained by the perturbation in a waveguide, that are related by coupled differential equations. A fiber Bragg grating has periodic variations in refractive index, which acts as a perturbation, and as a result the mode coupling occurs. The grating type defines the mode coupling so the grating acts as an optical filter or coupler between the core and the cladding modes. The coupled mode approach is the most general case, and for complicated grating structures, involves the numerical solution for two coupled differential equations, since analytic solution is only possible for the uniform grating [31].

Wave propagation in optical fibers is analyzed by solving Maxwell's equations with appropriate boundary conditions. The solutions provide the basic field distributions of the bound and the radiation modes of the waveguide. The coupling between the core 
and the cladding modes will be considered for this work, with and without a tilt of the grating.

\subsection{Coupled-wave analysis}

Equations of CMT are usually derived with the assumption of two coupled modes. In this section the coupling coefficients between all cladding-core modes will be determined.

For the unperturbed dielectric medium, which is homogeneous in $z$-direction, the normal modes of propagation of the unperturbed structure can be written in the form [53]:

$$
\vec{E}_{v}(x, y, z)=\bar{e}_{v}(x, y) e^{-i \beta_{v} z}
$$

where $\beta_{v}$ is the propagation constant of the $v^{t h}$ mode, $v$ is the mode index.

Power can be exchanged between modes only in a perturbed waveguide. The divergence of the power cross-product can be defined as [54]:

$$
\nabla \cdot\left[E_{1}^{*} \times H+E \times H_{1}^{*}\right\rfloor=-i \omega \varepsilon_{0} \Delta \varepsilon(x, y, z) E \cdot E_{1}^{*}
$$

where $E_{l}$ and $H_{1}$ are the fields of the unperturbed waveguide, and $\varepsilon(x, y, z)=\varepsilon(x, y)+\Delta \varepsilon(x, y, z)$ is the permittivity distribution function of a perturbed waveguide.

Integrating Eg. 3.2 over the entire waveguide cross section:

$$
\int \nabla_{t} \cdot\left[E_{1}^{*} \times H+E \times H_{1}^{*}\right], d s+\int \frac{d}{d z}\left[\left(E_{1}^{*} \times H+E \times H_{1}^{*}\right)_{t} \cdot \hat{z}\right] d s=-i \omega \varepsilon_{0} \int \Delta \varepsilon(x, y, z) E \cdot E_{1}^{*} d s
$$

" $t$ " in subscript represents the transverse components of the vectors. With twodimensional divergence theorem Eq. 3.3 reduces to: 
$\int \frac{d}{d z}\left[\left(E_{1, t}^{*} \times H_{t}+E_{t} \times H_{1}^{*}\right) \cdot \hat{z}\right] d s=-i \omega \varepsilon_{0} \int \Delta \varepsilon(x, y, z) E \cdot E_{1}^{*} d s$

Any transverse field component can be expanded in terms of modes:

$$
\begin{aligned}
& E_{t}=\sum_{v} a_{v}(z) \bar{e}_{v t} e^{-i \beta_{v} z} \\
& H_{t}=\sum_{v} a_{v}(z) \vec{h}_{v t} e^{-i \beta_{v} z}
\end{aligned}
$$

Though the longitudinal field component of the electric field has to be treated differently [54]:

$$
E_{z}=\sum_{v} \frac{\varepsilon(x, y)}{\varepsilon(x, y)+\Delta \varepsilon(x, y, z)} a_{v}(z) e_{v z} e^{-i \beta_{v} z}
$$

Thus, the fields of the perturbed waveguide are:

$$
\begin{aligned}
& E=\sum_{v} a_{v}(z)\left[\bar{e}_{v t}+\hat{z} \frac{\varepsilon(x, y)}{\varepsilon(x, y)+\Delta \varepsilon(x, y, z)} e_{v z}\right] e^{-i \beta_{v} z} \\
& H=\sum_{v} a_{v}(z)\left[\vec{h}_{v t}+\hat{z} h_{v z}\right] e^{-i \beta_{v} z}
\end{aligned}
$$

Now consider a mode $\mu$ travelling through the guided-wave grating. In the following chapter, in case of vectorial fiber modes, $\mu$ will be replaced with $l m . E_{l}$ and $H_{l}$ in terms of $\mu$ are going to be:

$$
\begin{aligned}
& E_{1}=\left(\vec{e}_{\mu t}+\hat{z} e_{\mu z}\right) e^{-i \beta_{\mu} z} \\
& H_{1}=\left(\vec{h}_{\mu t}+\hat{z} h_{\mu z}\right) e^{-i \beta_{\mu} z}
\end{aligned}
$$

Before going any further it should be noted that the modes are normalized by the time-averaged pointing vector:

$$
P_{z}=\frac{1}{2} \int \operatorname{Re}\left(E_{v}(x, y, z) \times H_{\mu}(x, y, z)\right) d s=\frac{1}{4} \int\left[\vec{e}_{v t}(x, y) \times \vec{h}_{\mu t}^{*}(x, y)+\vec{e}_{\mu t}^{*}(x, y) \times \vec{h}_{v t}(x, y)\right] \cdot \hat{z} d s=\delta_{v \mu}
$$


and is equal to 1 if $\beta_{v}=\beta_{\mu}>0$ and zero if $\beta_{\nu} \neq \beta_{\mu}$.

Next step would be to substitute Eqs. 3.7, 3.8 and 3.9(a,b) into Eq.3.4:

The left hand side of the Eq. 3.4 becomes:

$$
\left(E_{1}^{*} \times H_{t}+E_{t} \times H_{1}^{*}\right) \cdot \hat{z}=\sum_{v} a_{v}(z) e^{i\left(\beta_{\mu}-\beta_{v}\right) z}\left[\vec{e}_{\mu t}^{*} \times \vec{h}_{v t}+\vec{e}_{v t} \times \bar{h}_{\mu t}^{*}\right] \cdot \hat{z}
$$

Now integrating this expression keeping in mind Eq. 3.10, left hand side of the Eq.

\section{4 becomes:}

$$
\int \frac{d}{d z}\left\{\sum_{v} a_{v}(z) e^{i\left(\beta_{\mu}-\beta_{v}\right) z}\left[\vec{e}_{\mu t}^{*} \times \bar{h}_{v t}+\vec{e}_{v t} \times \vec{h}_{\mu t}^{*}\right] \cdot \hat{z}\right\} d s=\frac{d}{d z} \sum_{v}\left\{a_{v}(z) e^{i\left(\beta_{\mu}-\beta_{v}\right) z} 4 \delta_{v \mu}\right\}=4 \frac{d}{d z} a_{\mu}(z)
$$

Assuming that the mode is the forward propagating mode.

The right hand side of the Eq. 3.4 is:

$-i \omega \varepsilon_{0} \int \Delta \varepsilon(x, y, z) E \cdot E_{1}^{*} d s=-i \omega \varepsilon_{0} \sum_{v} a_{v}(z) e^{i\left(\beta_{\mu}-\beta_{v}\right) z} \int \Delta \varepsilon(x, y, z)\left[\vec{e}_{v t} \cdot \vec{e}_{\mu t}^{*}+\frac{\varepsilon(x, y)}{\varepsilon(x, y)+\Delta \varepsilon(x, y, z)} e_{v z} e_{\mu z}^{*}\right] d i$

Combining Eqs. 3.13 and 3.14:

$\frac{d}{d z} a_{\mu}(z)=-i \sum_{v}\left[\kappa_{v \mu}^{t}(z)+\kappa_{v \mu}^{z}(z)\right] e^{i\left(\beta_{\mu}-\beta_{v}\right) z} a_{v}(z)$

where

$$
\begin{aligned}
& \kappa_{v \mu}^{t}(z)=\frac{\omega \varepsilon_{0}}{4} \int \Delta \varepsilon \vec{e}_{v t} \cdot \vec{e}_{\mu t}{ }^{*} d x d y \\
& \kappa_{v \mu}^{z}(z)=\frac{\omega \varepsilon_{0}}{4} \int \frac{\varepsilon(x, y)}{\varepsilon(x, y)+\Delta \varepsilon(x, y, z)} e_{v z} e_{\mu z}^{*} d x d y
\end{aligned}
$$

are the coupling coefficients of the grating-assisted interaction of the transverse and longitudinal field components [54]. 
Since the longitudinal fields of the fiber modes are very small in comparison with the transverse ones, the longitudinal component of coupling coefficient $\kappa_{v \mu}^{z}(z)$ is much smaller than the transverse component, so the longitudinal component can be easily neglected [33]. And in the further calculations, the coupling coefficient will be denoted as $\kappa$.

Eq. 3.15 describes the general case of mode coupling due to a periodic dielectric perturbation. In reality, only the coupling between two modes is involved. Consider a simple Bragg-grating structure such as a single-mode slab or a narrow-band optical filter (Figure 3.1). Suppose a mode of unit amplitude and effective index $n_{\text {eff }}$ propagates in positive $z$ direction through a grating of length $L$, period $\Lambda$ and coupling coefficient $\kappa$.

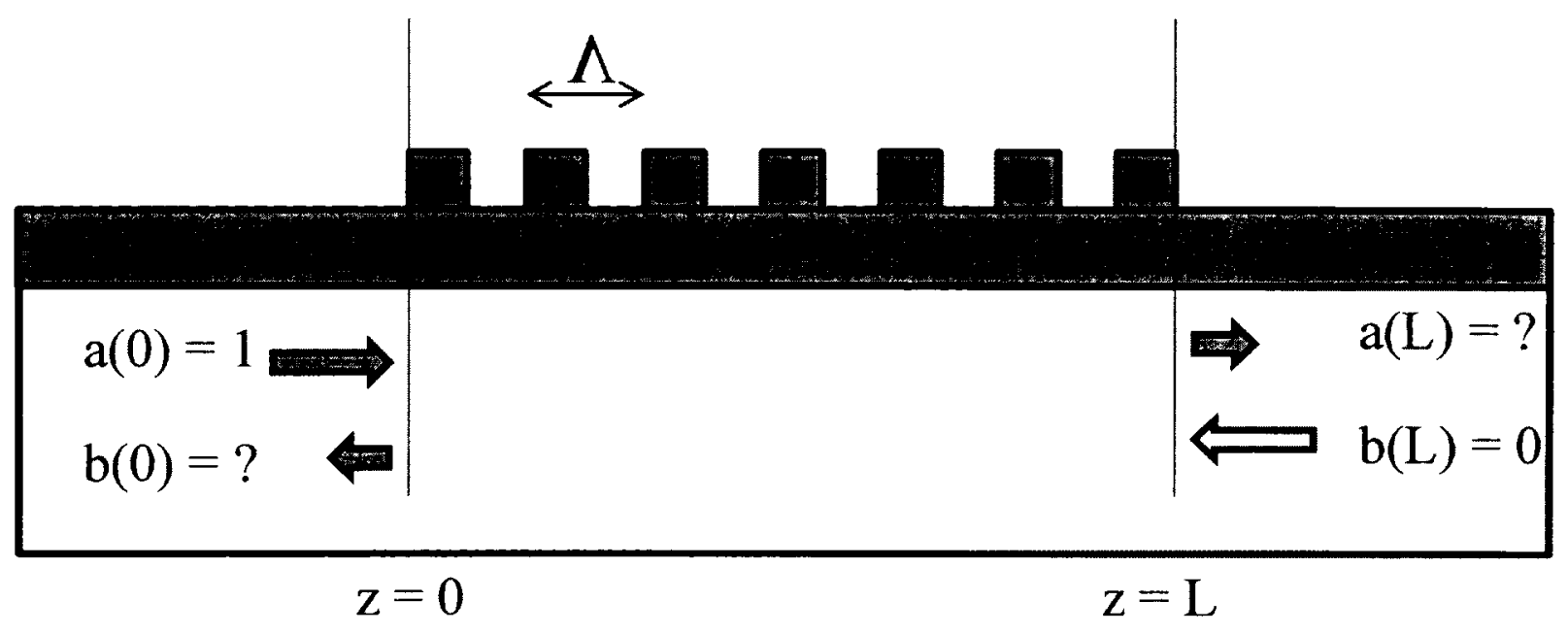

Figure 3.1: Slab waveguide grating structure.

The reflected and transmitted power can be studied, taking into account that two coupled modes are propagating in the opposite directions. The modes propagating in $+z$ and $-z$ directions can be labeled as $a(z), \beta_{v}>0 \Rightarrow \beta$ and $b(z), \beta_{v}<0 \Rightarrow-\beta$ respectively. Also let's define the Bragg parameter: 


$$
\delta=\beta-\frac{K}{2}
$$

Since the narrow-band reflection filter centered at the Bragg wavelength (Eq. 2.7) $\lambda_{B}=2 n_{e f f} \Lambda$ and $\beta=\frac{2 \pi n_{e f f}}{\lambda}$, the Bragg parameter can be written as [55]:

$$
\delta=\frac{2 \pi n_{e f f}}{\lambda}-\frac{\pi}{2 \Lambda}=2 \pi n_{e f f}\left(\frac{1}{\lambda}-\frac{1}{\lambda_{B}}\right)
$$

Then Eq. 3.15 can be split into two equations:

$$
\begin{aligned}
& \frac{d}{d z} a(z)=-i \kappa b(z) e^{i 2 \delta z} \\
& \frac{d}{d z} b(z)=i \kappa^{*} b(z) e^{i 2 \delta z}
\end{aligned}
$$

The solutions for these two differential equations with boundary conditions $a(0)=1$ and $b(L)=0$ can be written as:

$$
\begin{aligned}
& a(z)=\frac{\sigma \cosh [\sigma(L-z)]+i \delta \sinh [\sigma(L-z)]}{\sigma \cosh (\sigma L)+i \delta \sinh (\sigma L)} \\
& b(z)=\frac{-i \kappa^{*} \sinh [\sigma(L-z)]}{\sigma \cosh (\sigma L)+i \delta \sinh (\sigma L)}
\end{aligned}
$$

where $\sigma^{2}=\kappa^{2}-\delta^{2}$.

The reflection coefficient and the transmission coefficient of the grating reflector of length $L$ can be found as:

$$
\begin{aligned}
& R=b(0)=\frac{-i \kappa \sinh (\sigma L)}{\sigma \cosh (\sigma L)+i \delta \sinh (\sigma L)} \\
& T=a(L)=\frac{\sigma}{\sigma \cosh (\sigma L)+i \delta \sinh (\sigma L)}
\end{aligned}
$$

The power reflection and transmission coefficients, also known as reflectance and transmittance are 


$$
\begin{aligned}
& |R|^{2} \equiv|b(0)|^{2}=\frac{\kappa^{2} \sinh ^{2}(\sigma L)}{\sigma^{2}+\kappa^{2} \sinh ^{2}(\sigma L)} \\
& |T|^{2} \equiv|a(L)|^{2}=\frac{\sigma^{2}}{\sigma^{2}+\kappa^{2} \sinh ^{2}(\sigma L)}
\end{aligned}
$$

Eqs.3.20 a and $\mathrm{b}$ hold inside the filter stopband $\left(\delta^{2}<\kappa^{2}\right)$; in the oscillatory regions outside $\left(\delta^{2}>\kappa^{2}\right) \sigma$ becomes purely imaginary and the hyperbolic functions change into trigonometric ones [55].

It can be seen from these equations that the coupling efficiency decreases as the $\delta$ increases. The transmittance and reflectance are plotted as functions of wavelength for $\kappa L \approx 3.1$.

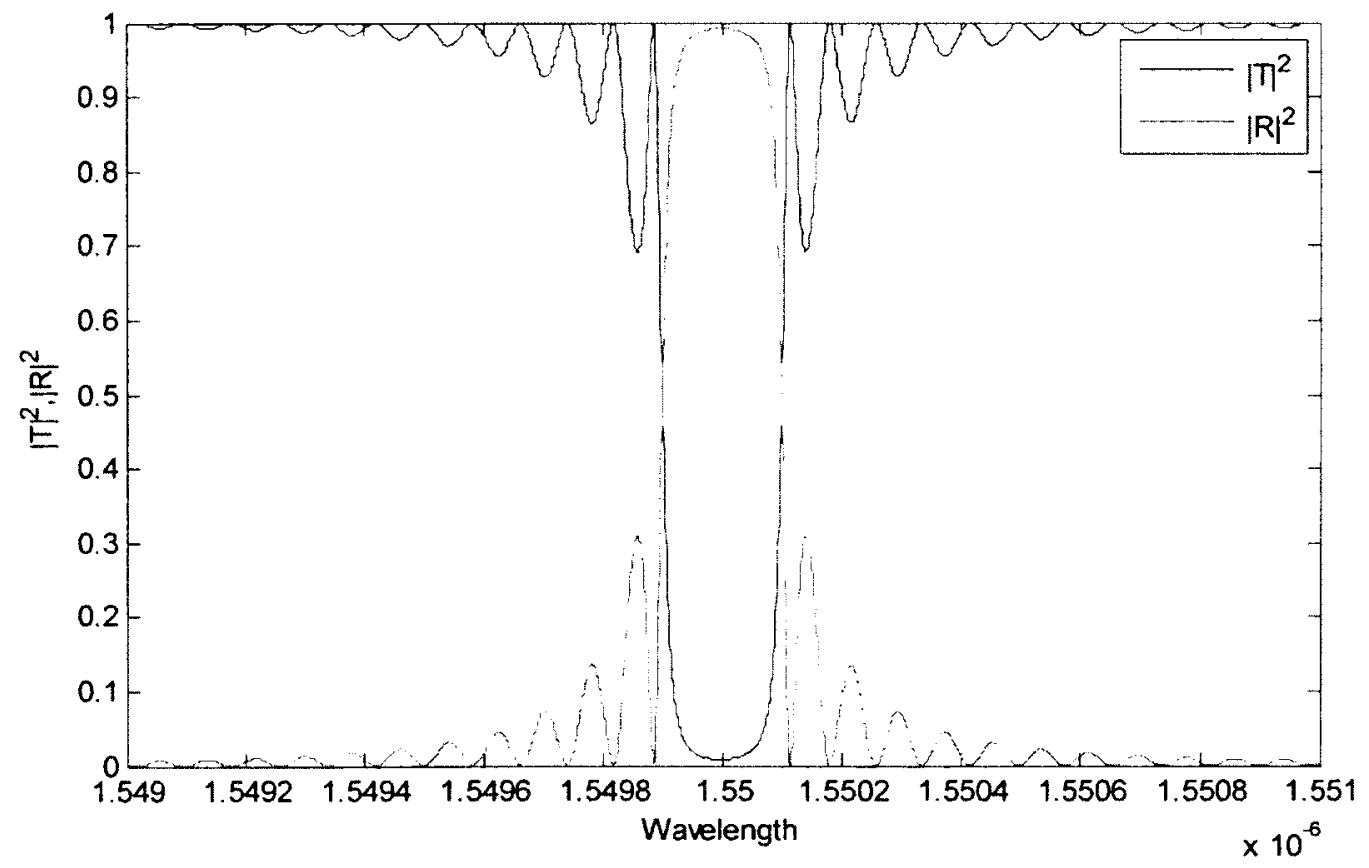

Figure 3.2: Example of reflectance and transmittance of grating reflectors. (Grating length $1 \mathrm{~cm}$, radius of the core of the fiber $1.8 \mu \mathrm{m}, n_{\text {core }}=1.47, n_{\text {clad }}=1.457, \Delta n=0.0003$ )

It can be seen from Figure 3.2 that the total power is conserved; power conservation also can be derived from Eqs. $3.22(\mathrm{a}, \mathrm{b}):|R|^{2}+|T|^{2}=1$. From Figure 3.2 it can also be 
noted that the power reflection coefficient reaches its peak when the Bragg condition ( $\delta=0)$ is met. From Eq. 3.22a the peak reflectance is:

$$
|R|_{\text {max }}^{2}=\tanh ^{2}(\kappa L)
$$

\subsection{Coupled-wave analysis for TFBGs}

Tilted fiber Bragg gratings are gratings with grating planes tilted at a small angle relatively to the $x$ - or $y$ - axis (Fig. 3.3). TFBGs couple light both to the backward propagating core modes and the cladding modes [38]. The resonant wavelengths for these mode couplings depend differentially on external perturbations. Using the core mode back reflection resonance as a reference wavelength, the relative shift of the cladding mode resonances can be used to selectively measure perturbations affecting the region outside the cladding independently of temperature.

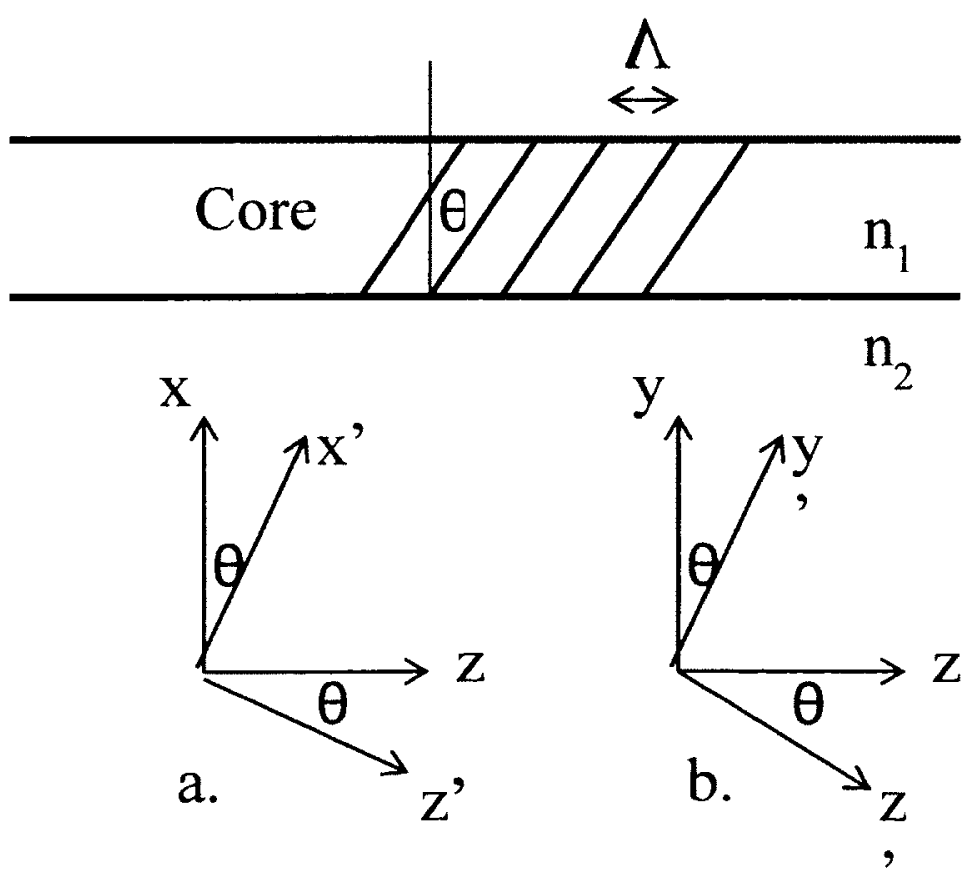


Figure 3.3: Diagram of the parameters associated with a TFBG. a. is the $x$-tilted and b. is the $y$-tilted grating.

Figure 3.3 demonstrates the tilted grating, whose planes are tilted around the $x$-axis (a) or y-axis (b) at an angle $\theta$.

The phase matching condition for the tilted grating can be re-written in a more convenient form for the resonance wavelength $\lambda_{r}$ of a resonance between the core mode and another mode labeled " $\mathrm{r}$ " [56]:

$\lambda_{r}=\left(N_{\text {eff }}^{\text {core }}\left(\lambda_{r}\right)+N_{\text {eff }}^{r}\left(\lambda_{r}\right)\right) \frac{\Lambda}{\cos \theta}$

Where $\Lambda$ is the period of the grating, $\theta$ is the tilt angle, $N_{\text {eff }}{ }^{\text {core }}$ is the effective index of the core mode and $N_{\text {eff }}{ }^{r}$ is the effective index of the mode $r$.

The dielectric perturbation for the tilted gratings, unlike for the untilted ones, depends on the tilt angle [17]:

$\Delta \varepsilon(x, y, z, \theta)=2 n_{0} \Delta n(x, y, z, \theta)$

where

$\Delta n(x, y, z, \theta)=\Delta n \cos \left(\frac{2 \pi}{\Lambda}(z \cos \theta+y \sin \theta)\right)$

Now the coupling coefficient for the tilted grating can be calculated by substituting equations (3.25) and (3.26) into equation (3.16):

$\kappa=\frac{\omega \varepsilon_{0}}{2} n_{0} \Delta n \int \vec{E}_{\text {core }}^{*} \vec{E}_{r} \cos \left(\frac{2 \pi}{\Lambda}(z \cos \theta+y \sin \theta)\right) d x d y$

From this equation it can be seen that the tilt angle determines the strength of the mode coupling. 


\section{Chapter: Transmission characteristics of fibers}

\subsection{Classification and properties of modes in three-layer fibers}

The basic structure of a fiber is the core surrounded by a cladding. Sometimes a metal coating is covering the cladding, which significantly changes the transmission characteristics, but for simplicity, it will not be considered in this section. To keep the analysis as clear as possible the simple three-layer, step-index fiber geometry with perfect circular symmetry (Fig. 4.1) will be considered:

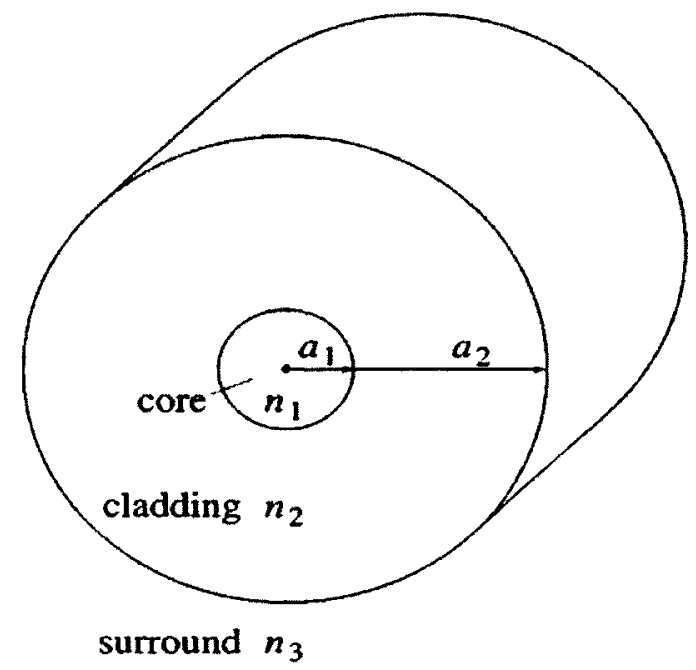

Figure 4.1: Schematic drawing of a cross section of a three layer fiber with various refractive indexes and different radii of layers.

In Figure 4.1 the inner cylinder is the core of the fiber with radius $a_{1}$ and outer shells are the cladding with radii $a_{i}$, the last shell usually represents the surrounding media. The refractive index of each layer is assumed to be smaller than that of a previous layer, thus the core will be of the highest refractive index. 
As previously assumed, the modes are propagating in the $z$-direction and can be described by the following equations $[56,57]$ :

$$
\begin{aligned}
& E_{l m}(x, y, z)=E_{l m}(x, y) e^{-i \beta_{l m} z} \\
& H_{l m}(x, y, z)=H_{l m}(x, y) e^{-i \beta_{l m} z}
\end{aligned}
$$

where $l=0,+-1,+-2, \ldots$ is the azimuthal mode number and $m=1,2, \ldots$ is the radial mode number. A mode can be also characterized with its effective refractive index $n_{e f f}=\frac{\beta_{l m} \lambda}{2 \pi}$, which depends on its propagation constant $\beta_{l m}(\lambda)$ and the wavelength $\lambda$.

The fields of modes with $l=0$ are symmetric and either purely azimuthally or radially polarized. The electric field of an azimuthally polarized mode is always parallel to a cylindrical surface. Thus the electric field has no $z$-component and such modes are transverse electric (TE). The same holds for the magnetic field of fully radially polarized modes, which are transverse magnetic (TM) [58]. In contrast, modes with $l>0$ are hybrid modes since the $z$-components of neither electric field nor magnetic field vanishes. They are classified $\mathrm{EH}$ or $\mathrm{HE}$ modes, depending on polarization of electric field relatively to the magnetic field. The mode with the highest effective refractive index $n_{\text {eff }}$ is the fundamental mode $\mathrm{HE}_{11}$ mode. All other $\mathrm{HE}$ and $\mathrm{EH}(l \neq 0)$ always come in neardegenerate pairs. For $l \geq 1$, there is a further exact degeneracy. Due to the rotational symmetry of the fiber, each hybrid mode $l m$ has a degenerate orthogonal counterpart whose fields are rotated by $\frac{\pi}{2 l}$. These are designated as "even" or "odd" modes respectively [57]. For the fundamental $\mathrm{HE}_{11}$ mode these terms correspond to the axis of polarization being along the $x$ - or $y$-axis, respectively. 
The step-index fiber (Fig. 4.1) is the most prominent fiber geometry, which consists of a core of refractive index $n_{1}$ and radius $a_{1}$, surrounded by a cladding of lower refractive index $n_{2}$ and radius $a_{2}$. The new parameters, generalized frequency $V$ and generalized guide index $b$, can now be defined as [54]:

$V=k a_{1} \sqrt{n_{1}^{2}-n_{2}^{2}}$

$b=\frac{N^{2}-n_{2}^{2}}{n_{1}^{2}-n_{2}^{2}}$

where $\lambda$ is the wavelength in free space and $N$ is the effective guide index such that $\beta=k N$.

If $V<2.4048$, the radial core fields have no root, thus only one mode can propagate. From Figure 4.2 it can be seen that all modes except $\mathrm{HE}_{11}$ mode are cut off if $V<2.4048$.

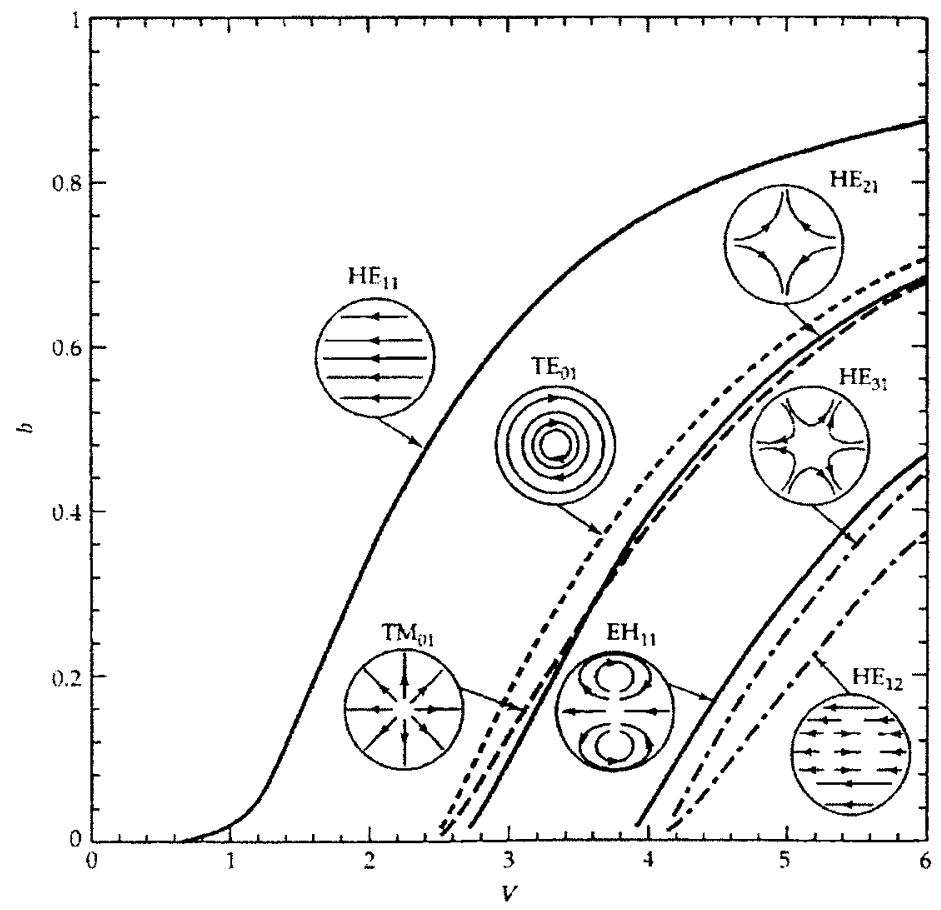

Figure 4.2: Dispersion of a fiber and electric field lines of some modes. [54] 


\subsection{Characteristic equations of modes}

Erdogan et al. [16] presented a full analytical solution of the three-layer structure which yields all hybrid modes with the azimuthal and radial integer indices $l$ and $m$. However this work is mainly focused on cladding mode reflections of conventional fiber gratings, for which only $l=1$ resonances occur. In 2011, Thomas et al. [59] looked at higher order modes with $l>1$. They used the notation employed by Erdogan, but expressed the azimuthal dependence in trigonometric rather than exponential form. In cylindrical coordinates $(r, \phi, z)$, the electric $\mathrm{E}$ and magnetic $\mathrm{H}$ fields of the cladding modes inside the core $\left(r<a_{1}\right)$ can be expressed in terms of Bessel functions $J_{n}$ of the first kind [59]:

$$
\begin{aligned}
& E_{z}=E_{l m} \frac{u^{2}}{\beta} P J_{l}(u, r) \sin (l \phi+\varphi) e^{i(\beta z-\omega t)} \\
& E_{r}=i E_{l m} \frac{u^{2}}{2}\left[(1-P) J_{l-1}(u, r)+(1+P) J_{l+1}(u, r)\right] \sin (l \phi+\varphi) e^{i(\beta z-\omega t)} \\
& E_{\phi}=i E_{l m} \frac{u^{2}}{2}\left[(1-P) J_{l-1}(u, r)-(1+P) J_{l+1}(u, r)\right] \cos (l \phi+\varphi) e^{i(\beta z-\omega t)} \\
& H_{z}=E_{l m} \frac{n_{e f f}}{Z_{0}} \frac{u^{2}}{\beta} J_{l}(u, r) \cos (l \phi+\varphi) e^{i(\beta z-\omega t)} \\
& H_{r}=i E_{l m} \frac{n_{e f f}}{Z_{0}} \frac{u}{2}\left[-\left(1-P \frac{n_{1}^{2}}{n_{e f f}^{2}}\right) J_{l-1}(u, r)+\left(1+P \frac{n_{1}^{2}}{n_{e f f}^{2}}\right) J_{l+1}(u, r)\right] \cos (l \phi+\varphi) e^{i(\beta=-\omega t)} \\
& H_{\phi}=-i E_{l m} \frac{n_{e f f}}{Z_{0}} \frac{u}{2}\left[-\left(1-P \frac{n_{1}^{2}}{n_{e f f}^{2}}\right) J_{l-1}(u, r)-\left(1+P \frac{n_{1}^{2}}{n_{e f f}^{2}}\right) J_{l+1}(u, r)\right] \sin (l \phi+\varphi) e^{i(\beta=-\omega t)}
\end{aligned}
$$

with the transverse wavevector $u=\frac{2 \pi}{\lambda} \sqrt{n_{1}^{2}-n_{e f f}^{2}}$. The constant $Z_{0}=\sqrt{\mu_{0} / \varepsilon_{0}} \approx 376.7 \Omega$ is the electromagnetic impedance in vacuum. The mode parameter $\mathrm{P}$ characterizes the relative strength of the longitudinal field components, and is used to classify modes as HE or EH. 
Note that Eqs. (4.4) represent two orthogonal sets of solutions, distinguished by the rotation angle $\phi$, which is $\phi=0$ for even modes or $\phi=-\pi / 2$ for odd modes. Thus, all hybrid mode solutions appear as degenerate pairs of fields with orthogonal polarization states.

\subsection{Modes in FBG and TFBG}

Consider a FBG that was inscribed in standard single mode fiber (SMF-28) with the light transmitted through the core with wavelength $\lambda=1.55 \mu \mathrm{m}$. It is possible to calculate the modes of this FBG with the waveguide solver FIMMWAVE by PhotonDesign, which will be described later.

There are number of typically observed intensity patterns for this grating is depicted in Figure 4.3.

The mode patterns can be classified as "rings" (Fig. 4.3(a)), "bow ties" (Fig. 4.3(b),(c)) and "quad ties" (Fig. 4.3(d),(e)). Figure 4.3 shows the intensities of various modes with different effective indexes of SMF-28 in air.

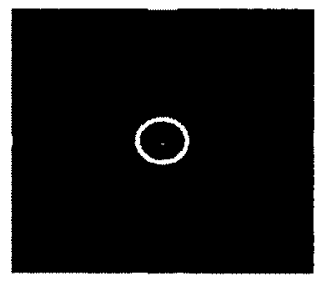

(a)

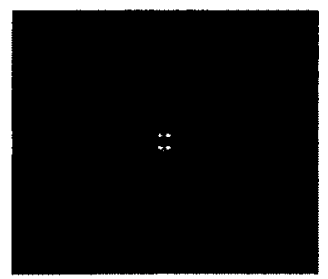

(b)

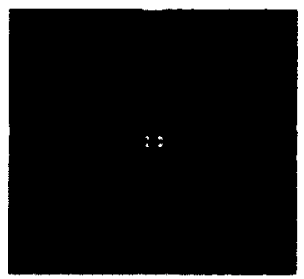

(c)

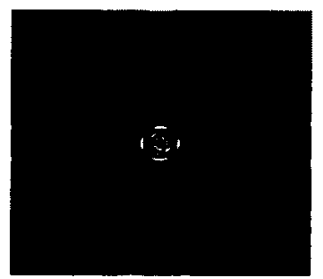

(d)

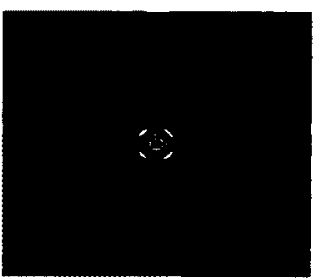

(e)

Figure 4.3: Typical mode patterns observed: (a) ring, (b) and (c) bow tie, (d) and (e) quad tie.

The HE and EH "bow-tie" are oriented $90^{\circ}$ to each other (Fig. 4.3 (b) and (c)). For the "bow-tie" modes guided by a grating the spatial orientations of the "bow-ties" in the doublet swaps if the polarization of the incident light is rotated by $90^{\circ}$. In that case the 
longer wavelength peak becomes the horizontal "bow-tie" and the shorter wavelength peak becomes the vertical "bow-tie" [28].

The grating index modulation has a well-defined orientation in space, which breaks the fiber's symmetry according to the tilt direction (assume the $y$-tilted). Thus there are two different cases where input electric field linear polarization is along $x$ (corresponding to $S$-polarized light incident on the grating planes) or $y$ ( $P$-polarized).

Then the scalar product between the electric field vectors in Eq. (3.27) reduces to a simple multiplication between $x$ - or $y$-polarized fields (depending on the polarization direction of the input mode). As a result, for a certain grating, the cladding modes for which Eq. (3.27) provides strong coupling will be different depending on the orientation of the input mode polarization relative to the tilt plane (because the $E_{x}$ and $E_{y}$ field components of a given cladding mode are quite different). Figure 4.4 shows an example of $E_{x}$ and $E_{y}$ components of the core mode and of a typical high order cladding mode. It is quite obvious from symmetry considerations that this cladding mode can only be strongly excited when the input core mode is S-polarized (i. e. along $x$ )[39]. 


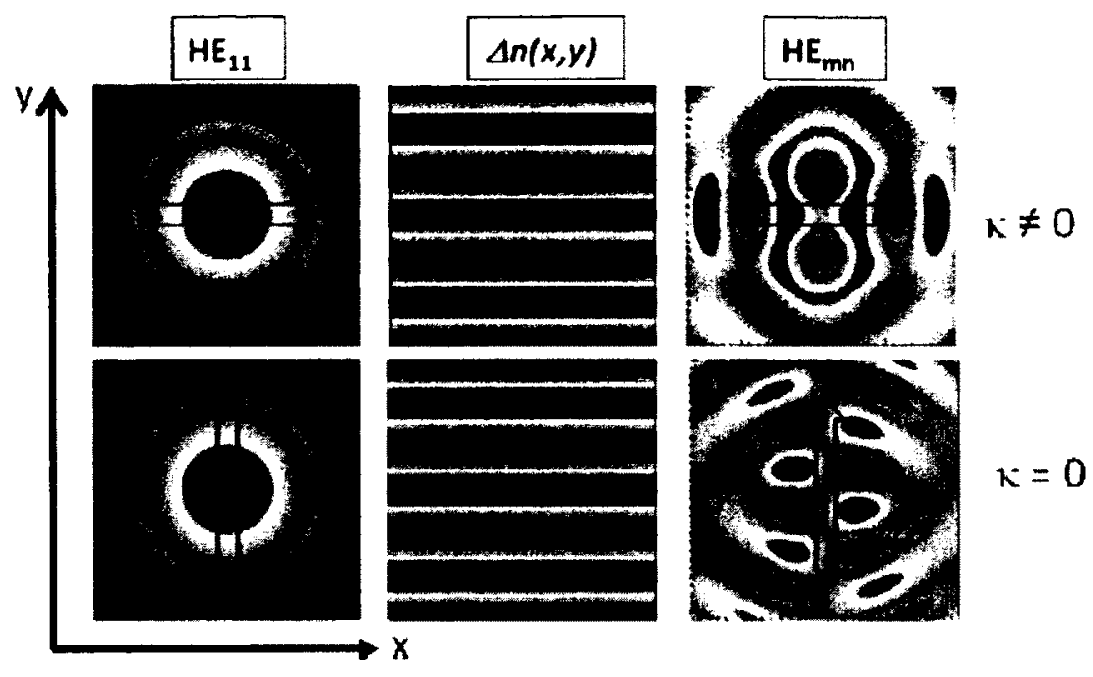

Figure 4.4: Three functions inside the integral for the coupling coefficient between the core mode $\left(\mathrm{HE}_{11}\right)$ and for a typical high order mode $\left(\mathrm{HE}_{\mathrm{mn}}\right)$, depending on the polarization of the input mode. The top row corresponds to S-polarized input light, and the bottom row to P-polarized light [39]. 


\section{Chapter: Software and Simulation Technique}

It is important to look at the different theoretical models that describe performance of Bragg gratings before conducting any experiments. It is essential to study these models, as each approach often offers a unique insight into physical mechanism of the grating-electric field interaction. There is a number of commercial software tools available that can be used to simulate and give a very accurate prediction for the FBGs behavior. The main challenge is dealing with the data, extracting it and then using it to calculate the transmission spectra. Suitable programming languages and software are very important in creating a model that is very close to real life experiment. The simulation program has to fulfill two requirements: the first is to provide the visual and user-friendly interface, so that the user can use it without any programming knowledge and obtain data by just changing the parameters. The other one is that the software has to provide a user with an amount of data that would be enough to build an accurate model. Results presented in this thesis were obtained using Matlab (2010b) as well as finite waveguide solver FIMMWAVE (v 5.3.8), commercial software offered by [Photon Design].

\subsection{FIMMWAVE}

FIMMWAVE is software that uses finite element method and can be applied towards modeling of light propagation within a variety of 2-dimentional and 3dimentioanal waveguide structures. It includes a variety of user interfaces that allows one to build an accurate prototype of different waveguides. It also provides different mode 
solvers to model these structures, such as numerical method or semi-analytical. Thus it enables a user to choose the most efficient and accurate method for a design of choice.

FIMMWAVE has three different interfaces for optical waveguides: ridge waveguide (RWG), fiber waveguide (FWG) and mixed geometry waveguide (MWG). The RWG is typical for defining epitaxially grown structures. The FWG is used to build cylindrically symmetric waveguides such as optical fiber. The MWG is the most abstract tool, which allows designing almost anything including an assembly of primitive shapes $[60]$.

This software allows user to take full advantage of each mode solving methods. It also includes a fully automatic mode scanner/finder - the MOLAB (Mode List Auto Builder), which allows defining the modes manually. As it has been said there are two types of mode solvers: semi-analytical which includes film mode matching (FMM) solver [61], and numerical mode solver, which includes finite-difference method (FDM) and finite element method (FEM). Fimmwave also includes an effective index solver for quick modeling of low $\Delta$ nor 2D structures and a cylindrical coordinate solver for modeling structures like optical fibers, which will be used for this work [60].

Once the waveguide has been defined as described in the Chapter 2 of Fimmwave manual [60], the modes then can be found using MOLAB finder. It is capable of finding the modes with almost complete certainty in a given range. Once the mode list has been build, each mode can be examined assuming that found mode is the mode of interest and if it is accurately generated. The list of modes usually consists of pairs of modes (even and odd). Figure 5.1 shows a pair of one mode of a single mode fiber (SMF-28). The accuracy of a mode is determined by accuracy of the propagations constant and the 
accuracy of the profile. Calculating the largest overlap between different modes can be a good test for modal accuracy; this is also called modal orthogonality. Ideally it should be zero, in case of perfectly orthogonal modes. But in the reality the number should be kept as low as possible, acceptable values are between $10^{-4}$ and $10^{-3}$.
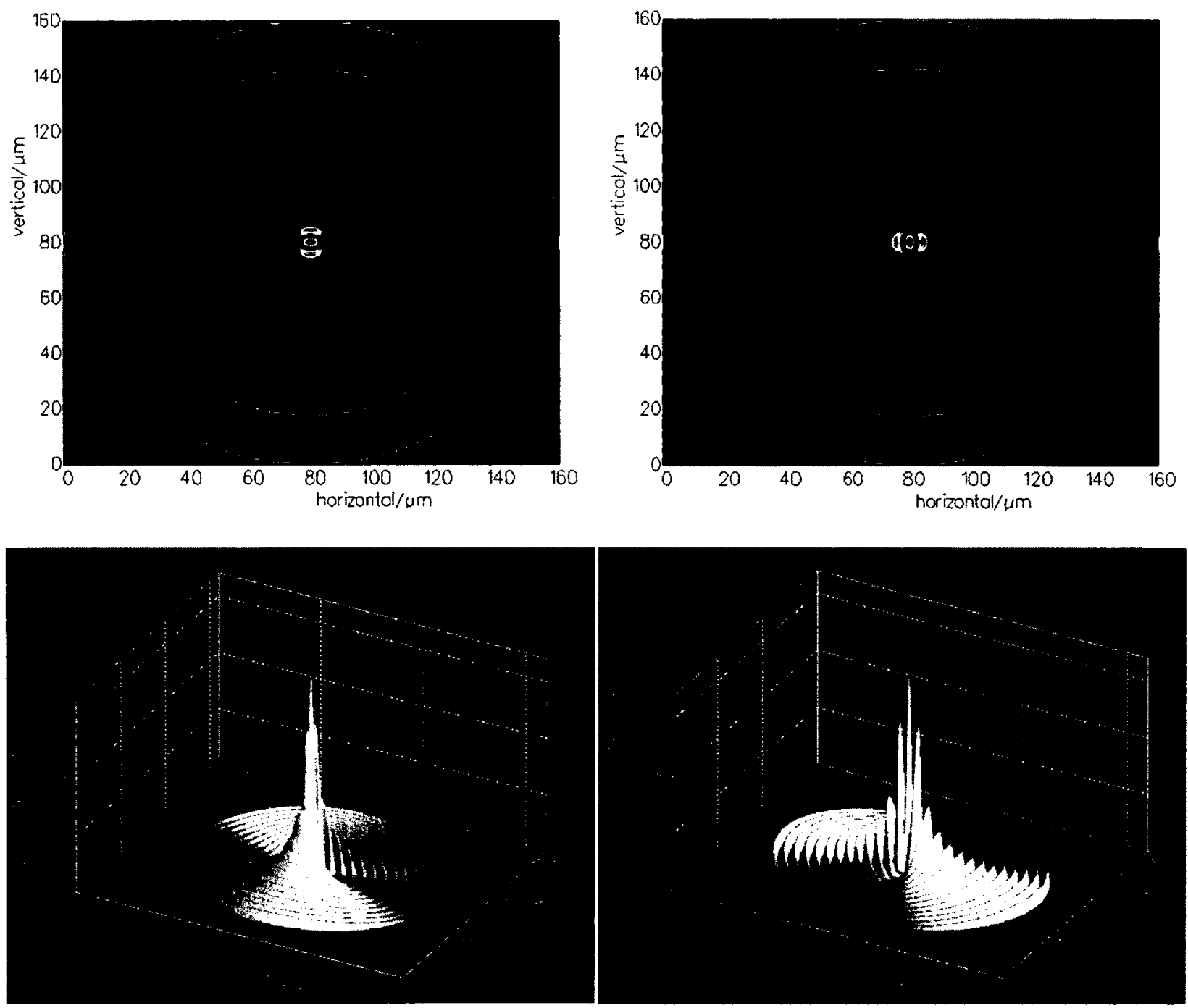

Figure 5.1: The intensity of even and odd components of one of the cladding modes of a fiber. Top row 2-

D and bottom row in 3-D. Intensity is measured in $\mathrm{n} J / \mathrm{m}^{3}$; the $x$ - and $y$-axes are in $\mu \mathrm{m}$. 
When it comes down to the mode amplitudes, Fimmwave gives already normalized modes, so that the power density is $1 \mathrm{~mW} / \mathrm{m}^{2}$. To be more precise, referring to Chapter 4.4 of the Fimmwave manual, resultant modes have the following property:

TEfrac $=\frac{\int E_{x} \cdot H_{y} d s}{\int P_{z}(s) d s}$

TEfrac is the fraction of the pointing vector with horizontal electric field and the flux $\int P_{z}(s) d s$ integrated over the surface is $I W$. Since the modes are already normalized, it won't be necessary to normalize them. And after the extraction of data the fields can be used as is to perform necessary calculations using other programming language such as $\mathrm{C}++$ or MatLab. In this work MatLab will be used.

\subsection{MatLab}

MatlLab is a mathematically-oriented interpreter language [62]. It is mainly used for simulation calculations, but it can also be used as numerical and symbolic calculator, a programming language, a modeling and data analysis tool etc. It uses symbolic expressions to provide a general representation of mathematics. Relatively simple syntax makes it easy to learn and use. It also makes the debugging process easier and faster than in other programming languages. It is very simple to manipulate complex and matrix arithmetic, compared with other languages. With MatLab it is not necessary to do any additional work when using complex numbers and matrix algebra. Along with useful toolboxes MatLab has a lot of useful functions built in [63].

One of the limitations of MatLab is the simulation running speed and the flexibility of the graphic user interface (GUI) when working with a large project. In this work the 
simulation can be broken into smaller parts, which will increase the speed significantly and in terms of managing a large project, it will be divided into two parts.

Due to its features MatLab is a very useful tool for this particular project. It will be used to acquire and to analyze data from Fimmwave. There are two ways of obtaining the data from FIMMWAVE: first is to access FIMMWAVE from MatLab remotely, and the second one is manually save the data from FIMWAVE and open it later with MatLab. Both of these ways have their advantages and disadvantages.

Fimwave can be accessed remotely via TCP/IP. In order to achieve that fimmwave.exe has to be started with the -pt argument: -pt <port No $>$ [60] is the TCP/IP port number on which Fimmwave should communicate with MatLab. Both Fimmwave and MatLab will connect to the host machine via this port. Thus MatLab can then control Fimmwave. MatLab interface connected to Fimmwave has been designed to be relatively simple while being very powerful - most commands pass through one central function, which can accept any arbitrary MatLab expressions. It then automatically returns any data from already running Fimmwave project as appropriate types (strings, real or complex numbers or arrays etc.). The disadvantage of this approach is it requires a lot from the operating system. The machine that operates this must be very powerful, has lots of memory; on the other hand, the simulation can be running with almost no manual work. Though a user will have to make sure that the modes that are being used are the correct ones, so some manual work still requires in order to use the correct modes for the calculations.

For this work a computer with Windows 7 Professional (C) 2009) with Intel $\mathbb{B}$ Core $^{\mathrm{TM}} 2 \mathrm{CPU} @ 2.40 \mathrm{GHz}$, with $3 \mathrm{~GB}$ of RAM and 64-bit operating system was used. In 
order to achieve relatively high accuracy when calculating the coupling coefficient, the matrixes of the fields required to be as big as possible. It was deduced, that $100 \times 100$ complex matrix is sufficient to get an accurate result. For this matrix size the amount of RAM was not enough to run the simulation with the above described data acquisition.

Another way to acquire data from Fimmwave is to run the project in Fimmwave, then save the data for each mode in the MatLab folder on a disc and then use this data in MatLab for further calculations. In this way only the modes of interest will be used, the mode list can be polished, and the machine that is being used does not have to be very powerful, since Fimmwave can only work on a single core. 


\section{Chapter: Modeling of the Tilted Fiber Bragg Grating and Results}

\subsection{Building a Fiber Waveguide and Finding its modes}

Fiber waveguide (FWG) can be built in Fimmwave as described in Section 2.2 of a user manual [60]. The FWG node type is used to describe a circularly symmetric waveguide. One of the building steps of a FWG is the type of a fiber, in this project. As it was mentioned previously, the step-index type (stepped) is going be chosen. The refractive index profile $n(r)$ varies in discrete steps; the user defines the refractive index of each step or a layer (Figure 6.1). In this project the thickness as well as the refractive index (material) of each layer will be chosen as follows:

\begin{tabular}{lrl}
\hline Layer & thickness $(\mu m)$ & material \\
\hline Core & 4.15 & $\mathrm{GeO}_{2}-\mathrm{SiO}_{2}$ (concentration 0.03) \\
Cladding & 58.35 & $\mathrm{SiO}_{2}$ \\
Surrounding media & 20 & Varies from 1 to $\sim n_{\text {eff }}$ of a mode
\end{tabular}

Table 6.1: Layers of a bare fiber and the properties of each layer 

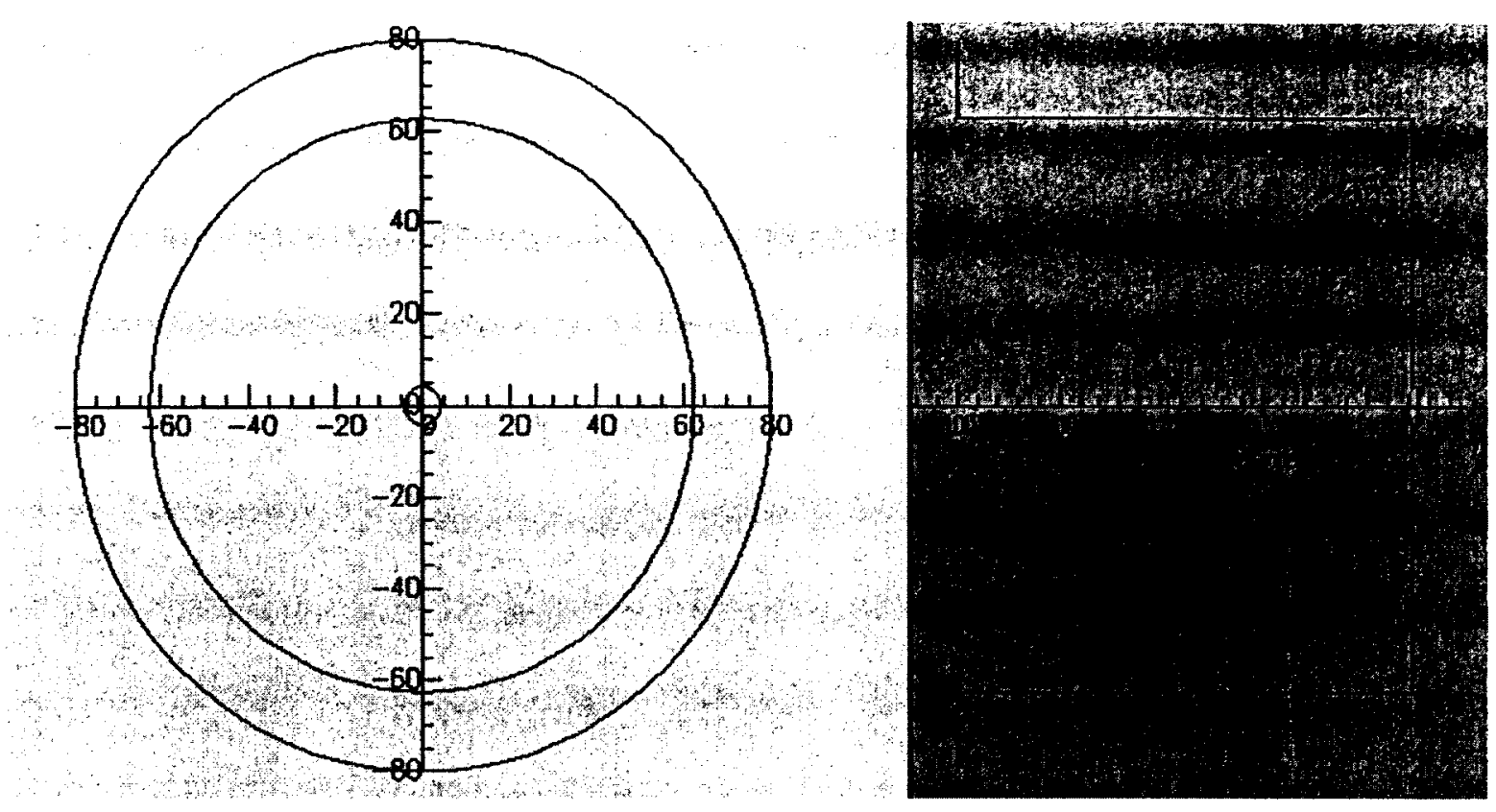

Figure 6.1: The cross section of chosen fiber (left) and its refractive index profile (right)

Now that the fiber has been build, it is possible to create a mode list using MOLAB single mode fiber (FDM) complex solver. This solver implements a full-vectorial finitedifference method and allows one to accurately model waveguides with high-step refractive index profiles, slanting/curved interfaces and gradient profiles. The $F D M$ Solver models both real and lossy materials and anisotropic dielectric tensors (diagonal tensor).

User has to specify the azimuthal quantum number, $m$-order (which was identified as $l$-number in previous chapters) and the polarization number $p$-order (which was identified as $m$-number in previous chapters) in the solver parameters. $m$-order follows the conventional indexing of the cylindrical modes so that the fundamental mode (the fastest propagating mode) $\mathrm{HE}_{11}$ is given by $m$-order $=1$, not zero! Setting $\mathrm{m}$-order $=0$ gives the $\mathrm{TE}_{0 \mathrm{~m}}, \mathrm{TM}_{0 \mathrm{~m}}$ modes. , zero indicates the mode, which is always pure TE-like or TM-like. The scalar approximation produces solutions that 2-fold or 4-fold degeneracy, for instance there are always 2 or 4 modes with the same effective index. For $m=0, p$ can 
be 1 or 2 , giving the fundamental modes that have either $E_{x}=0$ or $E_{y}=0$ - typically referred to as $\mathrm{HE}_{11}$ modes. The table 6.2 summarizes the different modes for different $m$ and $p$ values [60]:

\begin{tabular}{rlr}
\hline$m$-order $=$ & 0 \\
\hline$p$-order & Name & \\
1 & $\mathrm{TE}_{0 \mathrm{~m}}$ & \\
2 & $\mathrm{TM}_{0 \mathrm{~m}}$ & \\
\hline$m$-order $>$ & 0 & Notes \\
\hline$p$-order & Name & The modes appear in the list ordered \\
1 & odd $\mathrm{HE}_{l m}$ and odd $\mathrm{EH}_{l m}$ & as follows: $\mathrm{HE}_{l l}, \mathrm{EH}_{l l}, \mathrm{HE}_{l 2}, \mathrm{EH}_{l 2}$, etc. \\
\hline
\end{tabular}

Table 6.2: Modes with various $m$ - and $p$-vlues

It is simple to distinguish if the mode is even or odd, since it is defined by the $p$ number. In this work the even modes will be considered $(p=2)$. In addition, the field profiles are going to be taken only in the middle of the fiber, i.e. $x$ and $y$ are not going to be from 0 to $160 \mu \mathrm{m}$ as shown in Figure 5.1, but for better accuracy $x$ and $y$ components are going to be from 70 to $90 \mu \mathrm{m}$, since the important data is confined near the core region and closer to the cladding the values are nearly zero. The matrixes are still going to be $100 \times 100$ so that the resolution is higher for more precise result. Now each mode can be individually examined and the data for all field profiles can be extracted and saved. It is important to note that the modes are complex, i.e. each matrix value has real and imaginary parts.

Then this data can be used to perform necessary calculations in MatLab. Equations derived in Chapter 3 (coupled mode equations) will be used to calculate the transmission spectra for various refractive indexes of surrounding media. Luckily MatLab handles easily complex matrixes and it is easy to perform any calculations with this software. 
It then becomes a simple matter of putting necessary equations into the MatLab. The coupling coefficient will be calculated using Eq. 3.27. Then the transmission and reflection can be calculated using Eqs. $3.22 \mathrm{a}$ and $\mathrm{b}$ respectively. Then the transmission can be plotted as a function of wavelength, which can be calculated using the grating phase matching condition (Eq. 3.24). Results of such simulations will be presented in the following section.

\subsection{RESULTS}

\subsubsection{Bare SMF in different surrounding media}

Using the model described above, the modes can be built for cases when different media with corresponding refractive indexes surrounds fiber. Overall, the modes can be calculated for any range of effective index. The simplest example is the bare fiber surrounded the air $(n=1)$. As well as the effective index of each mode, TE component represents the fraction of the Poynting vector with horizontal electric field, i.e. the fraction of the Poynting vector given by $E_{x}$ and $H_{y}$ (Eq. 5.1). TE can be plotted as a function of wavelength that was calculated using Eq. 3.22: 


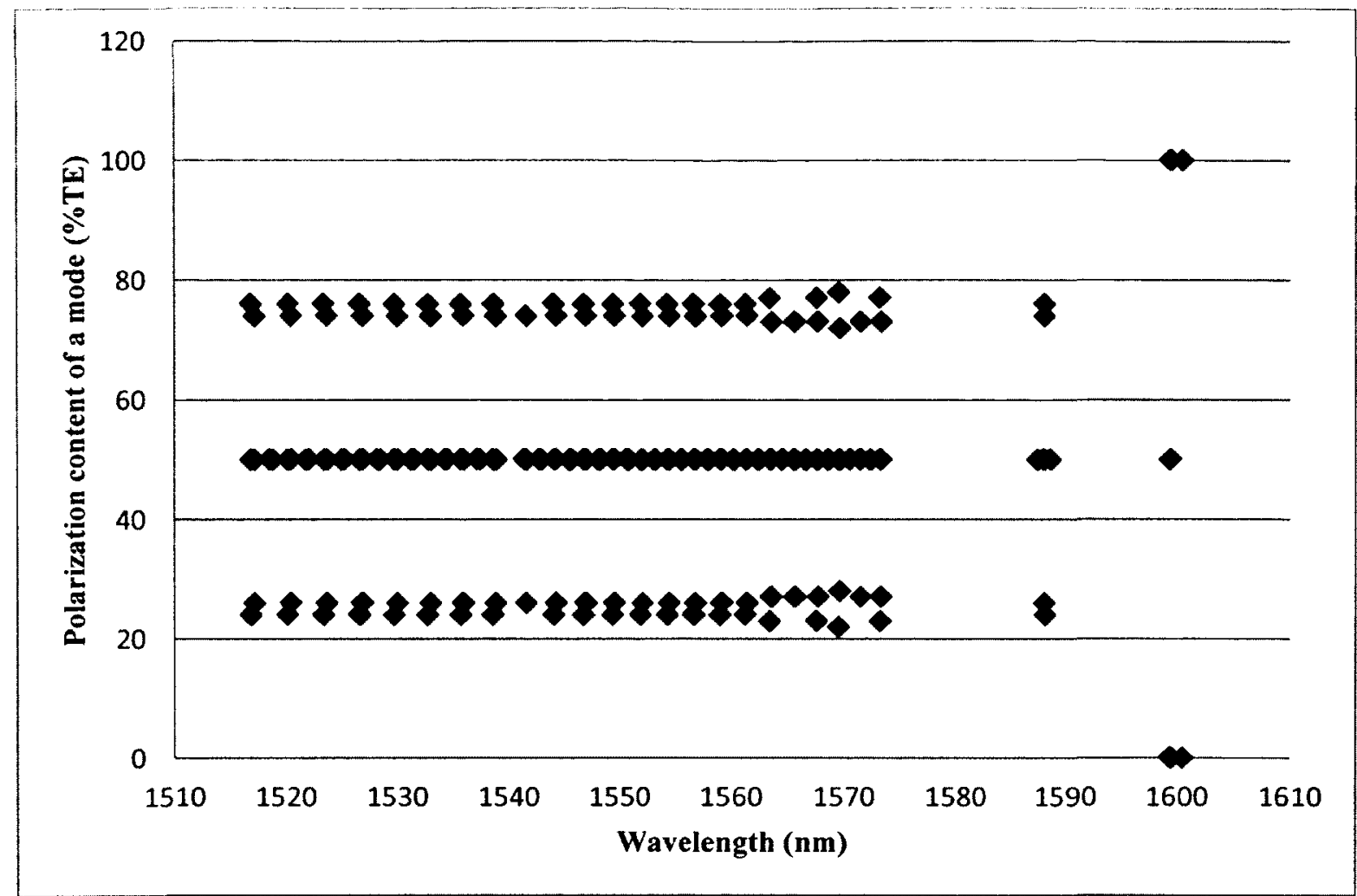

Figure 6.2: Polarization of found modes as function of resonance wavelength found for bare SMF-28 surrounded by air.

Similar plot can be generated for a fiber immersed in surrounding media, for example Figure 6.3 shows polarization components for fiber immersed in water $(n=1.315)$ 


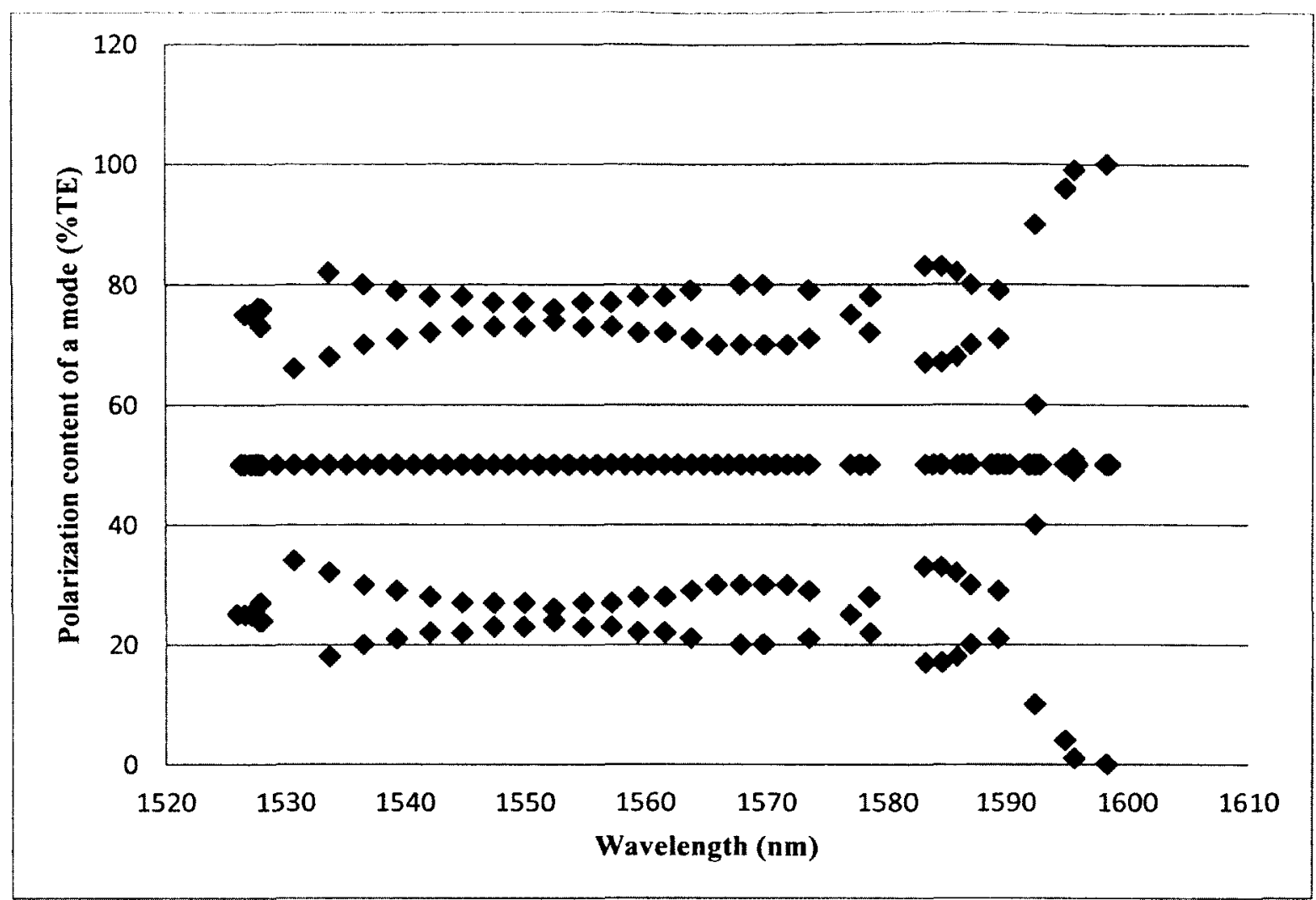

Figure 6.3: Polarization of found modes as a function of resonance wavelength found for bare SMF-28 immersed in water.

The data used for Figures 6.2 and 6.3 was directly transported from Fimmwave to Excel. The azimuthal number ( $m$-order) for the above figures was ranging from 0 to 3 ; and the polarization number ( $p$-order) was from 1 to 2 . From these figures it can be seen that the modes do pair up and the polarization content changes dramatically depending of the refractive index of the fibers' environment. Figure 6.3 shows the change in polarization of a mode as a function of wavelength, the change in polarization of modes as effective index of cladding modes increases and approaches the value of the effective index of the core mode can be observed. 


\subsubsection{Gold-coated SMF in different surrounding media}

This model can be extended to find modes propagating in a metal coated fiber. A thin $(0.05 \mu \mathrm{m})$ gold layer with complex refractive index of $(0.55-i 11.5)$ can be added between the cladding and the surrounding media. In practise certain amount of light is lost due to scattering and diffraction, so the simulation tool makes it as close to real case as possible. The complex part of the effective index of each mode can be attributed to the fact that modes in metal coated waveguide become lossy. It can be plotted as a function of wavelength calculated in the similar manner as in the previous example.

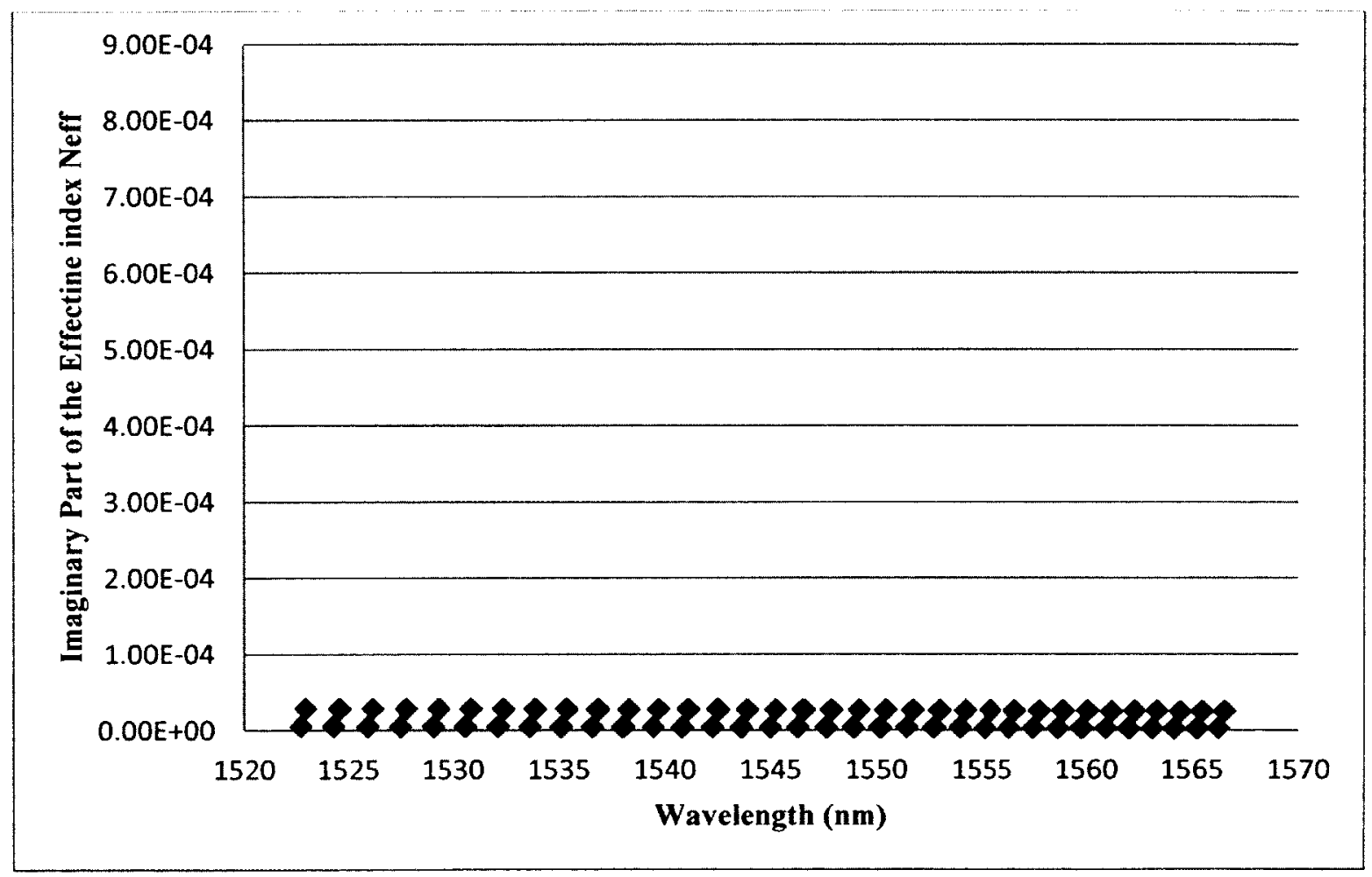

Figure 6.4: Imaginary part of modes propagating in Au-coated fiber as a function of wavelength; in air. 


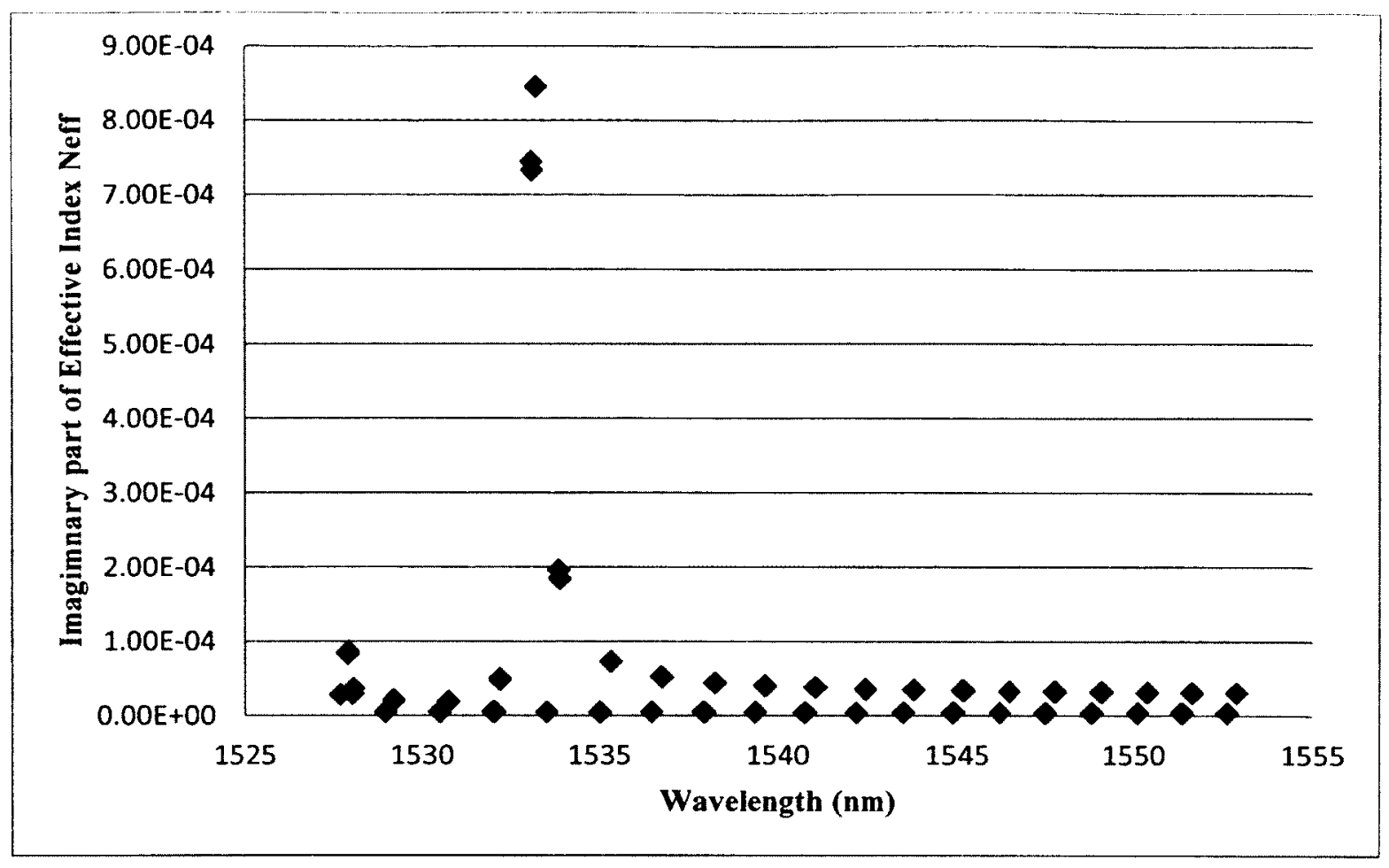

Figure 6.5: Imaginary part of modes propagating in Au-coated fiber as a function of wavelength; in water.

Figures 6.4 and 6.5 demonstrate once again the dramatic change in the response as refractive index of surrounding media changes. On Figure 6.5 there is a very big spike in the mode loss, which should correspond to excitation of the surface plasmon polariton (SPP). SPP is a non-radiative, surface electromagnetic wave that propagates at the boundary between mediums having dielectric constants of the opposite sign. Usually, one medium is dielectric while the other is metal or doped high-mobility semiconductors such as InSb [64] This phenomenon is explained in more details by Y. Shevechenko et al. [65]. In this work they explain the concept of surface plasmon polaritons and show that the tilted-grating-assisted excitation of surface plasmon polaritons on gold-coated singlemode optical fibers depends strongly on the state of polarization of the core-guided light. In particular, they demonstrate that when the external refractive index changes or when the surface of the gold is modified (by the addition of a biolayer for instance), the 
complex effective index of the plasmon changes and different cladding modes become attenuated. Their simulations show that the loss of a small subset of the cladding modes resonances increases sharply almost exactly at the location of the experimentally observed surface plasmon.

\subsubsection{Gold-coated TFBG in different surrounding media}

The transmission spectrum can be plotted for different wavelengths transmitted though a 10-degree grating to support the experimental results obtained by Y. Shevchenko et al [65]. The mode list was built for SMF and a cladding mode with effective index of 1.357039595 in air was chosen. This mode has $m$-order $=1$ and the $p$ order $=2$, the same as for core mode. The other modes, with $m$-order 3 and 5 and effective indexes close to the above value, are also used for better accuracy. Thus, three modes with very similar effective indexes and $m$-order $=1,3$ and 5 are taken, the three calculated values for the transmittance and wavelengths are averaged for every entry of wavelength of incoming wave. The transmission spectrum was calculated using Eq. 3.22

(b), where the parameters were chosen to be the following: tilt angle $10^{\circ}$, wavelength of an incoming wave is ranging $1.525 \ldots 1.555 \mu \mathrm{m}$, speed of light in vacuum $c_{0}=3.0 \times 10^{8} \mathrm{~m} / \mathrm{s}^{2}$, electrical permittivity $\varepsilon_{0}=8.85 \times 10^{-12} \mathrm{~F} / \mathrm{m}$. The transmission spectra were built (Fig. 6.6) for a surrounding refractive indexes of air $(n=1)$ and water $(n=1.315)$. 


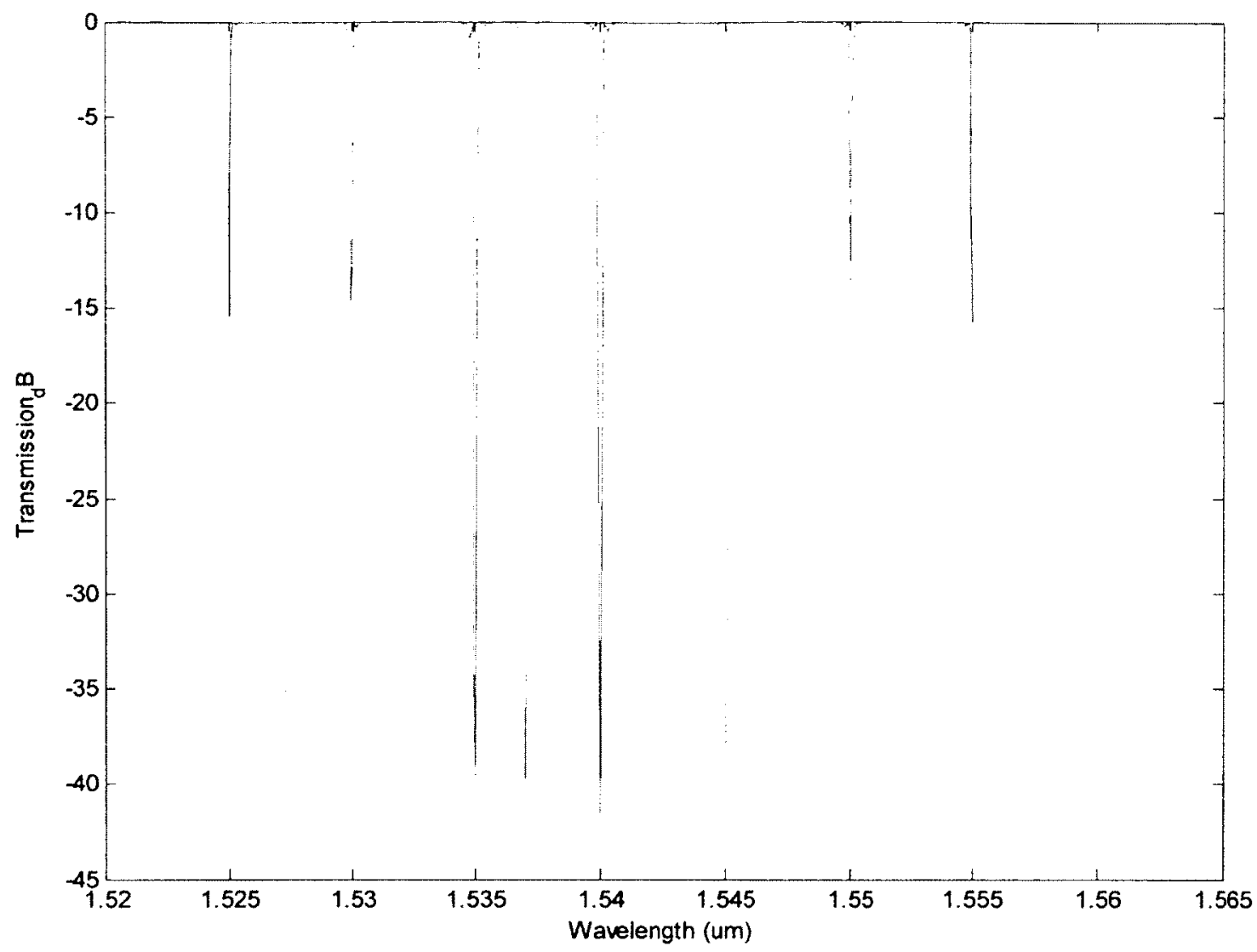

Figure 6.6: Transmission spectrum as a function of wavelength for the 10-degree grating surrounded by Air. 


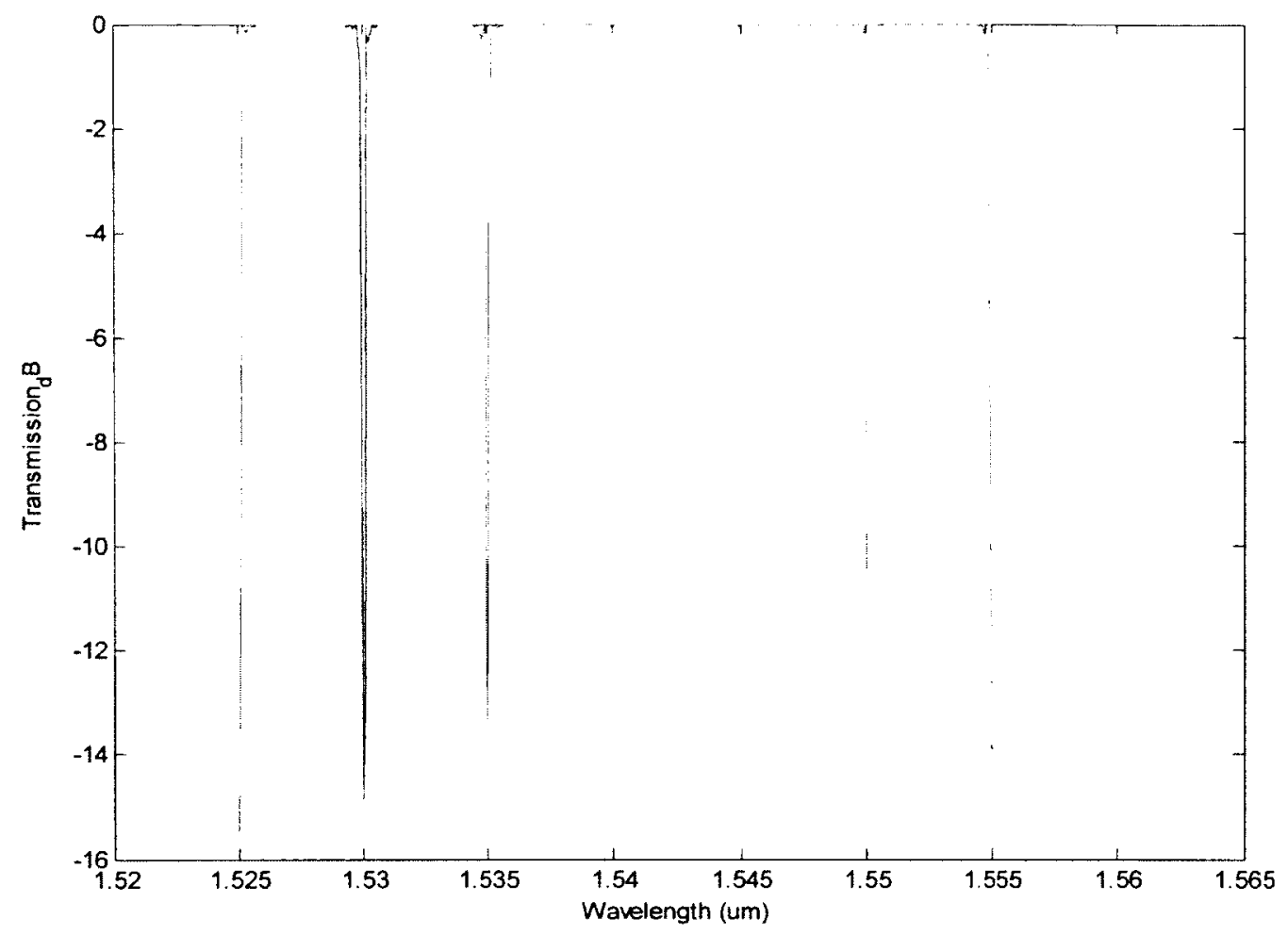

Figure 6.7: Transmission spectrum as a function of wavelength for the 10-degree grating surrounded by water.

Figures 6.6 and 6.7 can be compared to the experimental results obtained by Shevchenko et al. [65]. It can be seen that the transmission spectrum changes as the refractive index of surrounding media changes. As predicted in the previous section, on Fig. 6.7 there is a very big spike in the transmission, which corresponds to excitation of the surface plasmon polariton (SPP).

\subsubsection{Spectral response of bare TFBG immersed in different surrounding media}

Now the transmission spectra can be plotted for a certain cladding mode of a tilted fiber Bragg grating placed in various surrounding media. As it was described above, the mode list was built for SMF and a cladding mode with effective index of 1.353209 in air 
was chosen. This mode has $m$-order $=1$ and the $p$-order $=2$, the same as for core mode. The other modes, with $m$-order 3 and 5 and effective indexes close to the above value, are also used for better accuracy. As in the previous section, three modes with very similar effective indexes and $m$-order $=1,3$ and 5 are taken, the three calculated values for the transmittance and wavelengths are averaged for every refractive index of surrounding media change. The transmission spectrum was calculated using Eq. 3.22 (b), where the parameters were chosen to be the following: tilt angle $\theta=6^{\circ}$, wavelength of an incoming wave is $1.55 \mu \mathrm{m}$, speed of light in vacuum $c_{0}=3.0 \times 10^{8} \mathrm{~m} / \mathrm{s}^{2}$, electrical permittivity $\varepsilon_{0}=8.85 \times 10^{-12} \mathrm{~F} / \mathrm{m}$. The transmission spectra were built (Fig. 6.8) for a range of surrounding refractive indexes from 1 to the value of the effective index of a mode. This value is called cut off value, in this case it is 1.35 . 


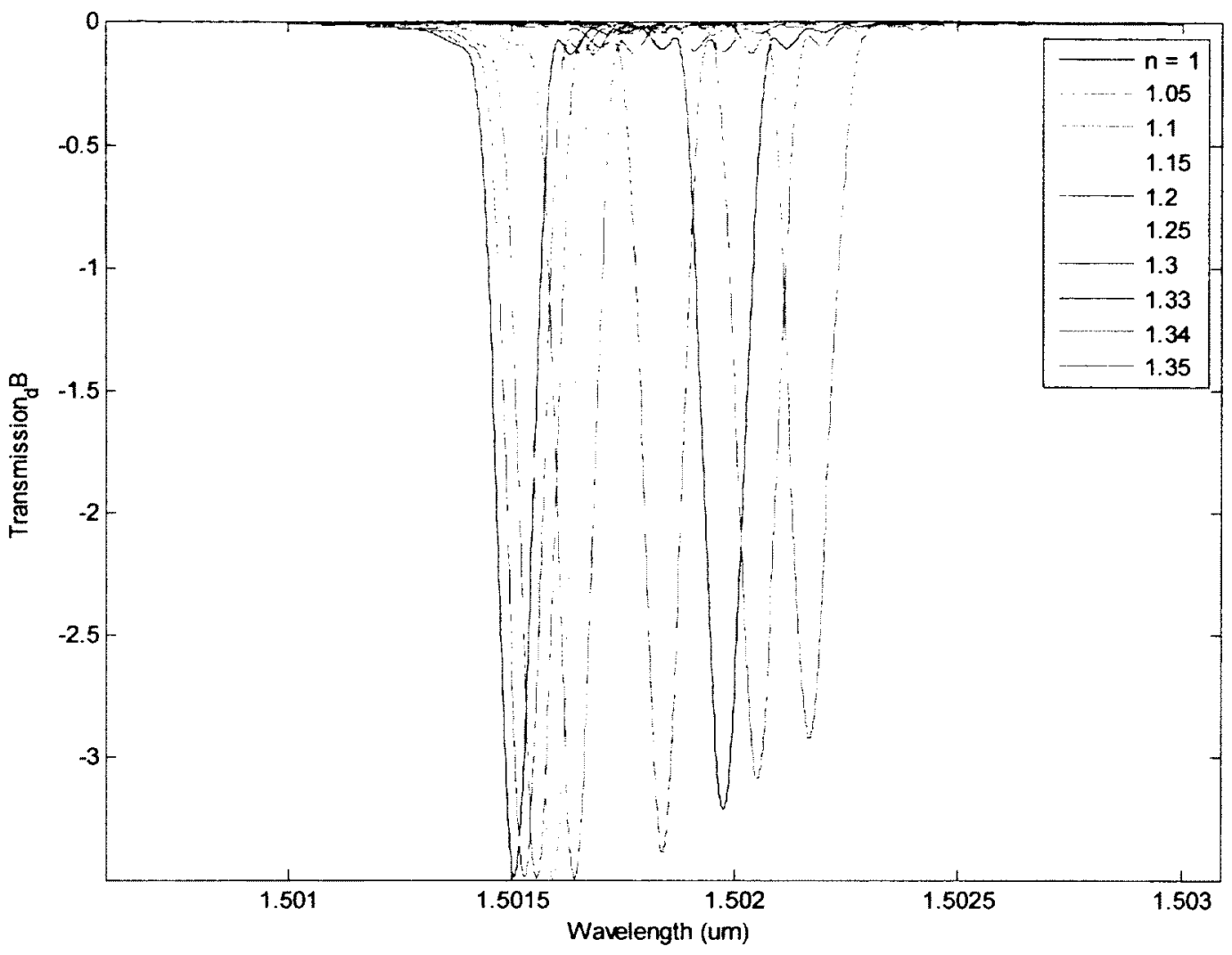

Figure 6.8: Transmission spectra as a function of wavelength for the surrounding refractive index $n_{\text {out }}$ changing from 1 to 1.35 . 


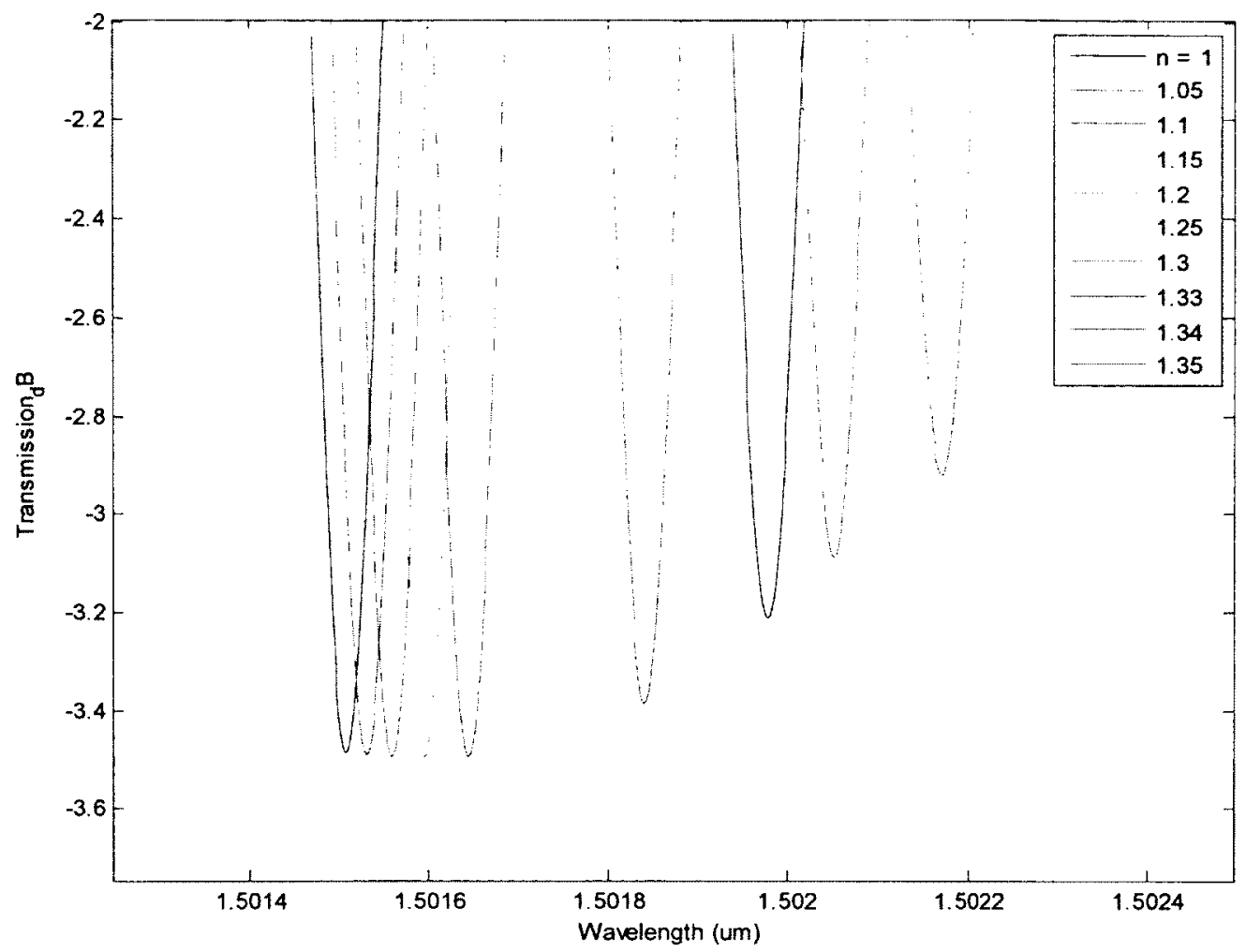

Figure 6.9: Same spectra as Fig. 6.8 but zoomed in.

Figure 6.9 shows data presented in Figure 6.8 but at a bigger scale.

In order to examine this plot each peak of this transmission spectrum was plotted as a function of wavelength: 


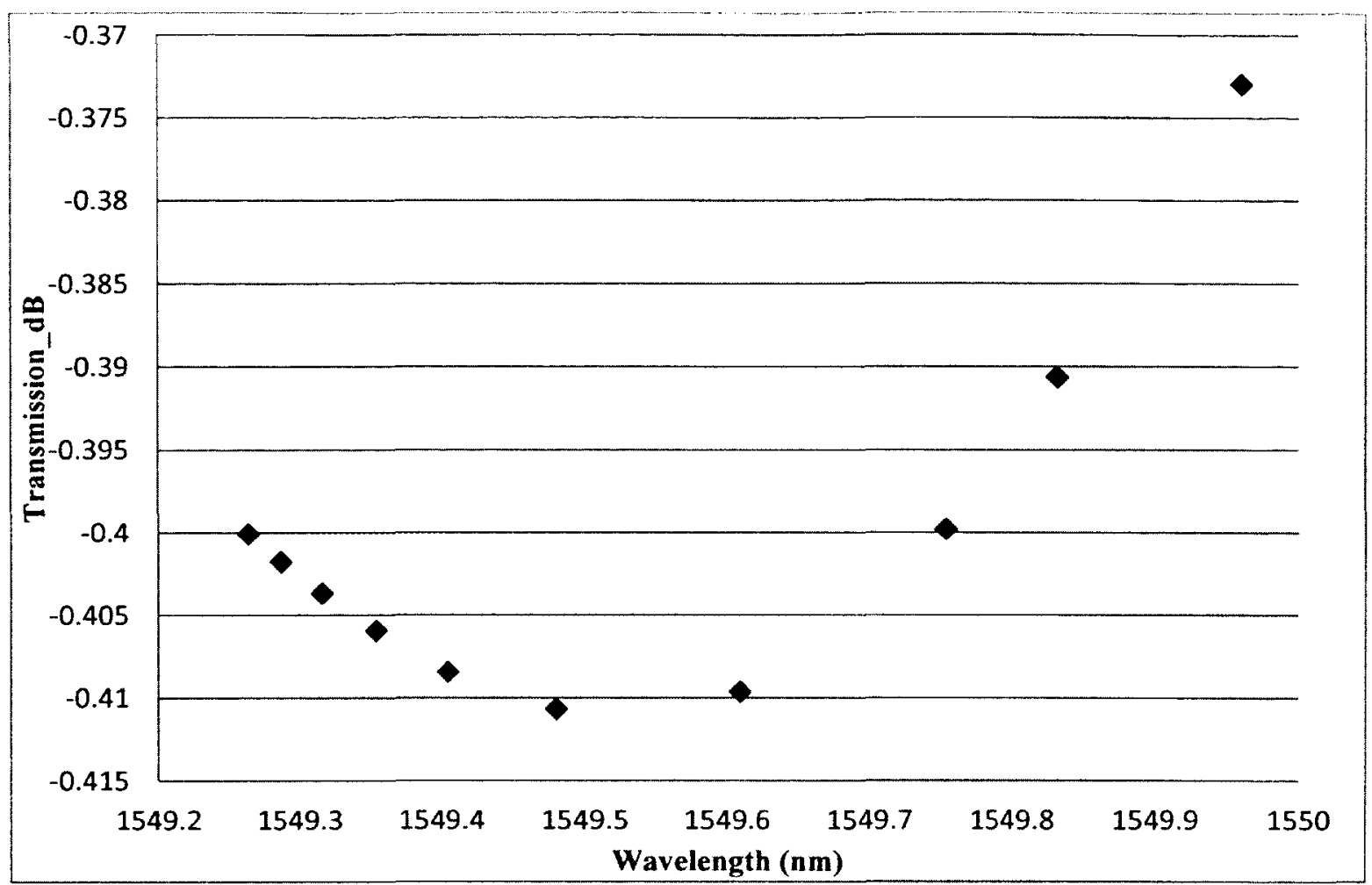

Figure 6.10: Transmission loss of a certain resonance as a function of wavelength.

The effect of effective index change can also be examined as the outside refractive index increases. From Figure 6.10 it can be seen that the effective index of a mode gradually increases as the outside refractive index $\left(n_{\text {out }}\right)$ increases. More dramatic increase is noticeable as $n_{\text {out }}$ approaches the cut off value. 


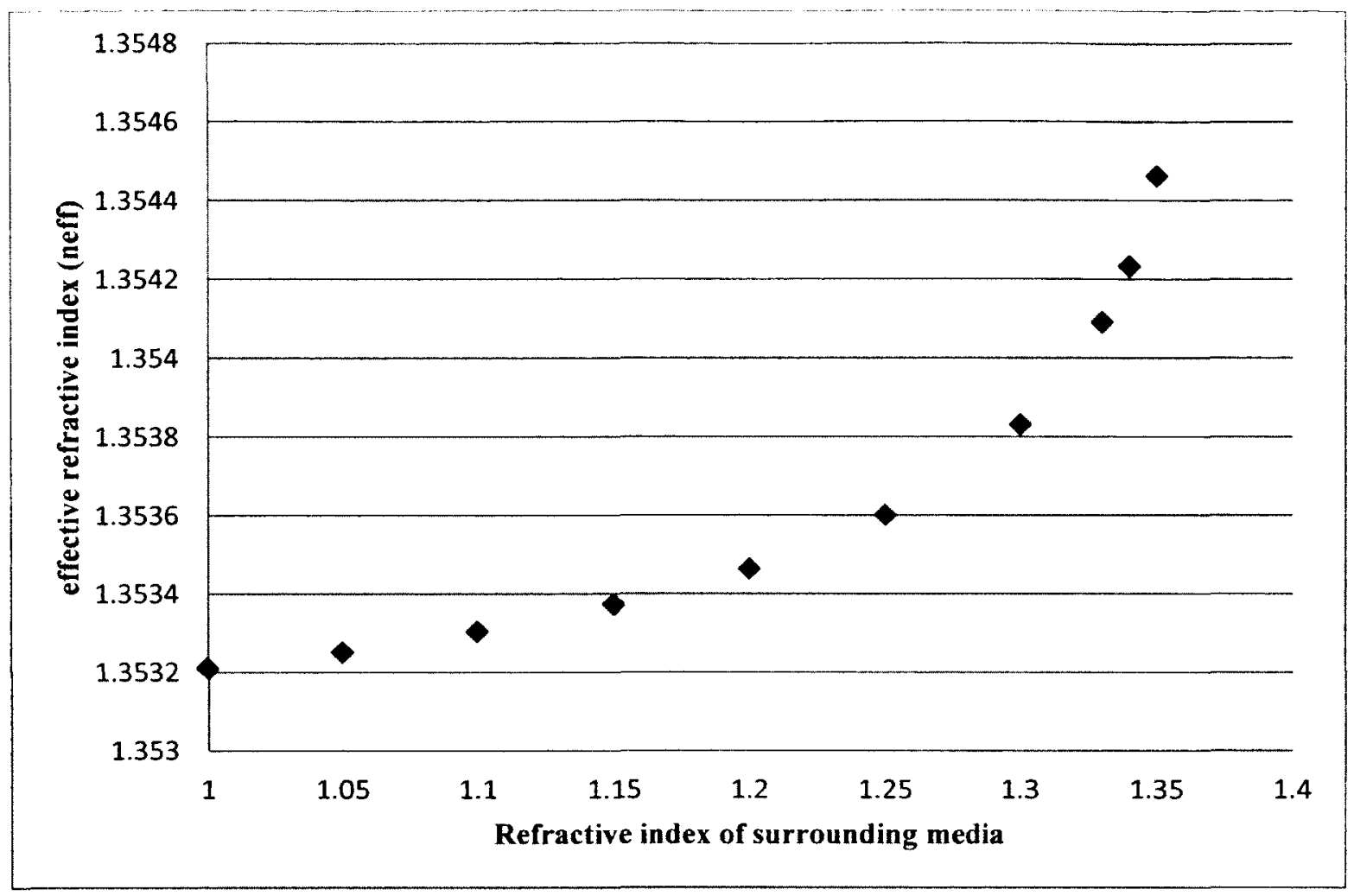

Figure 6.11: Effective index of a mode as a function of refractive index of surrounding media.

Now the simulation results can be compared to the experimental data that was obtained by Chan et al. [66]. Chan et al. examined short-period fiber Bragg gratings with weakly tilted grating planes and determined that multiple strong resonances in transmission were generated. The experimental results showed that the wavelength separation between selected resonances allows the measurement of the refractive index of the surrounding medium $\left(n_{\text {out }}\right)$ for values between 1.25 and 1.44 with an accuracy approaching $10^{-4}[66]$.

The results are for the measurements that were performed for the TFBG (tilt angle $=6^{\circ}$ ) immersed in sucrose solutions. Figure 6.12(a) shows an experimental grating transmission spectrum measured in air. For the same grating Figures 6.12(b) and 6.12(c) present overlapped spectra for several values of $n_{\text {out }}$ ranging from 1.377 to 1.43 . There is 
a notable shift of the hight-order cladding modes while lower-order modes and Bragg resonance are not affected. Then the relative resonances (with respect to $\lambda_{B}$ ) can be plotted as a function of refractive index of a surrounding media $\left(n_{\text {out }}\right)$.

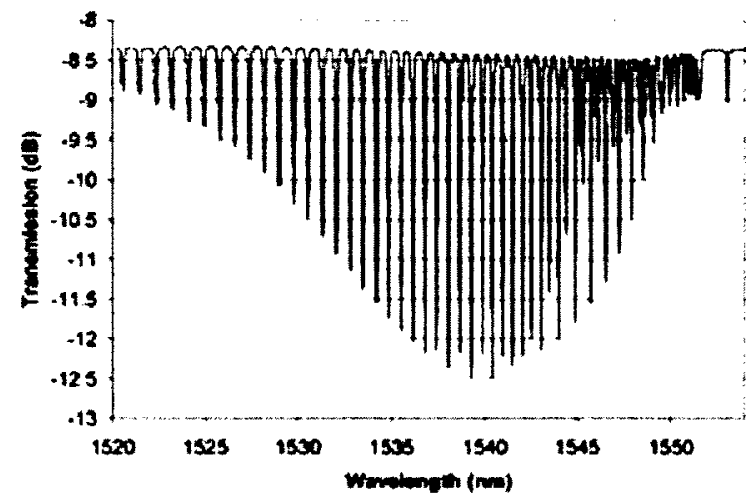

(a)

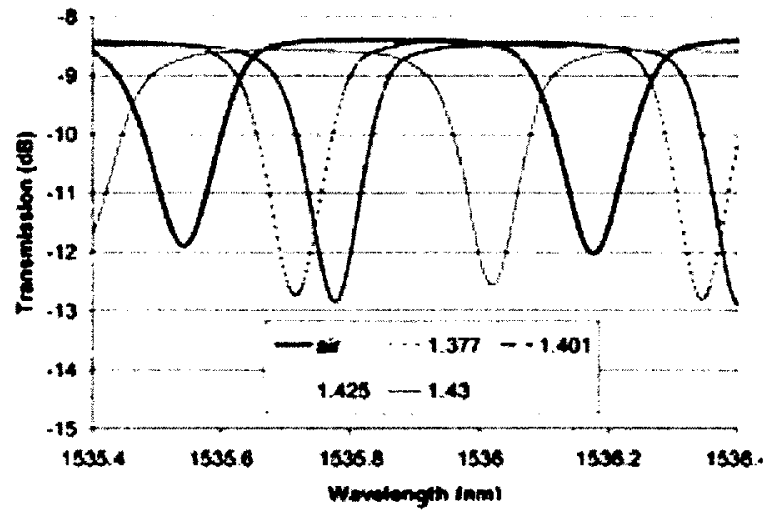

(c)

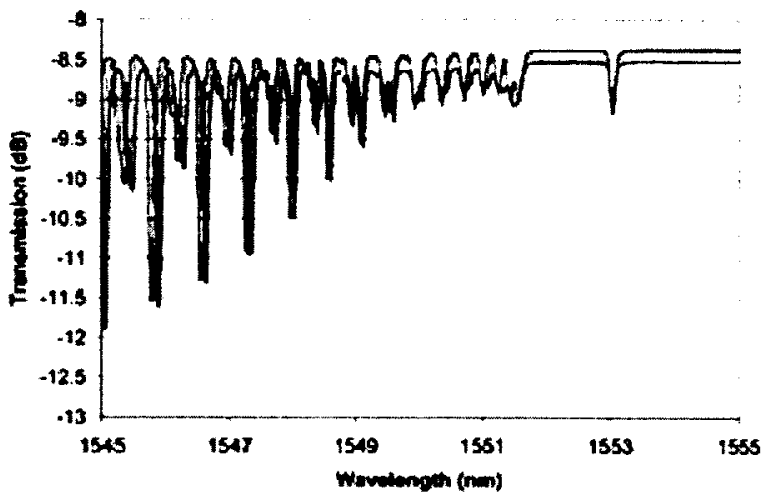

(b)

Figure 6.12: a). Typical experimental TFBG transmission spectrum (SMF-28 fiber, $\theta=6^{\circ}$ ) measured in air. b). Several measurements with various refractive indices of the outer medium near the Bragg resonance. (c) Same spectra as (b) but zooming in on a particular resonance near $1535.5 \mathrm{~nm}$ [66].

As it can be seen from Figure 6.9 the peaks of the transmission spectrum are shifted in the same manner as in Figure 6.12(c). The transmission "dip" decreases slightly as the refractive index of the outside medium increases and grows as the effective index of a mode approaches the value of the refractive index of surrounding media $\left(n_{\text {out }}\right)$. Comparing the theoretical model to the experimental results obtained by Chan et al. one 
can see that the shapes and the way the peaks shift are quite similar, meaning that the model was constructed correctly. The two figures (Fig. 6.9 and 6.12) were constructed with some differences in parameters. Thus, they can be compared only qualitatively.

\subsubsection{Spectral response of Gold-coated TFBG immersed in different surrounding media}

The next step will be plotting the transmission spectrum as a function of wavelength for the gold-coated tilted fiber Bragg grating. Nothing needs be changed in the MatLab code, but the gold layer needs to be added to the simulated structure in the same manner as it was performed in section 6.2.2.

The transmission peaks will be plotted as a function of wavelength:

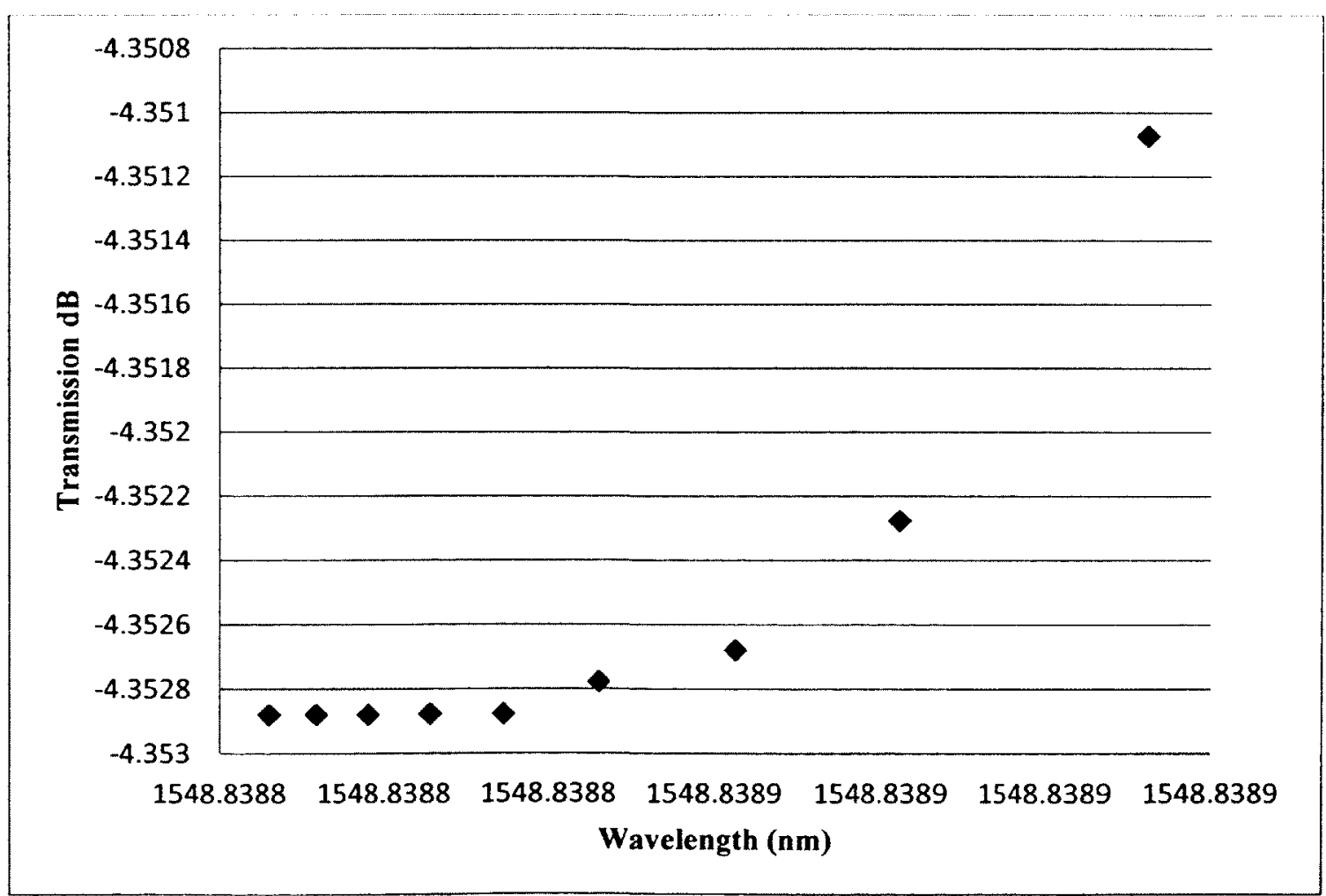

Figure 6.13: Tramsission loss of a modes propagating in a gold coated SMF-28. 


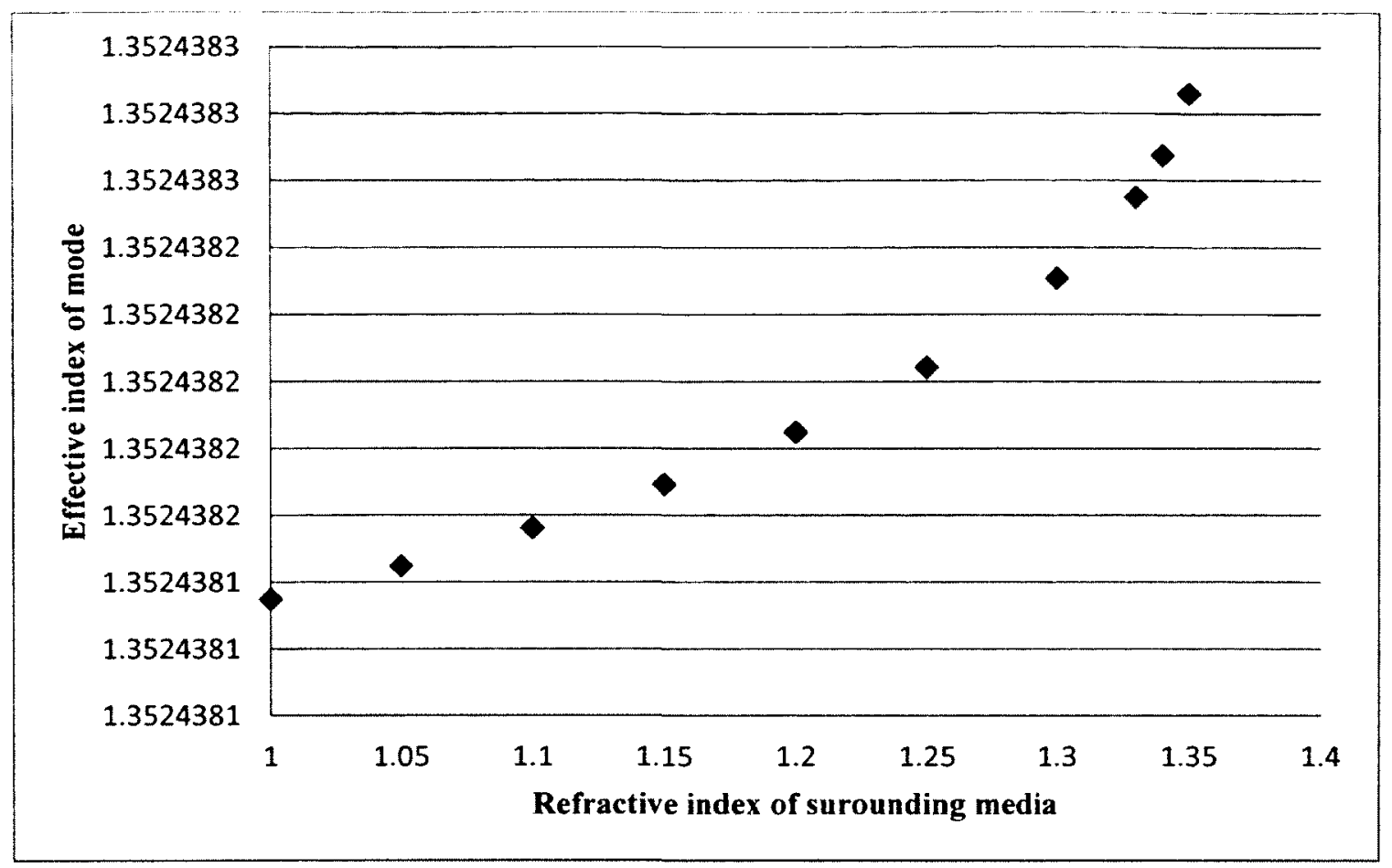

Figure 6.14: Effective index of a mode $\left(n_{\text {eff }} \approx 1.3528\right)$ as a function of refractive index of surrounding media.

By comparing the results obtained for bare TFBG with results for coated TFBG, it is quite clear that the Figure 6.13 is quite different from Figure 6.10. On Figure 6.13 the transmission "dip" does not decrease as the refractive index of surrounding media increases, but the value maintains the same; though as the $n_{\text {out }}$ approaches the cut off value, the peaks behave in the same manner as for the bare TFBG. If one takes a closer look at these figures, it can be noted that the effective index of the mode, hence the wavelength, does not change as dramatically for the gold-coated fiber as for the bare one. In the bare fiber case the effective index of mode changed from 1.353242 to 1.354501 ( $\left.\Delta n_{\text {eff }}=1.259 \times 10^{-3}\right)$ and in the coated fiber case, the effective index of a mode changed from 1.35243813 to $1.35243828\left(\Delta n_{e f f}=1.259 \times 10^{-7}\right)$. The same can be said about Figure 6.11 and Figure 6.14. The effective index of the mode changed significantly as it 
can be seen from Figure 6.11 and in Figure 6.14 for the gold-coated fiber; the change in effective index is very small, even though the refractive index of the surrounding media was changed identically in both cases. By comparing these figures quantitatively, one can see that the shapes of the plots are somewhat similar, though there is a noticeable discrepancy in the resultant values. These differences can be explained by the generation of the models using different parameters, i.e. the presence of the gold layer. 


\section{Chapter: Conclusions and Future Work}

The main goal of this project was to study the fiber Bragg gratings, and especially tilted fiber Bragg gratings. To develop a model that will allow studying the mode coupling within an optical fiber. Component modeling is a fundamental step in a process of designing of the fiber optical sensors and communication systems. Simulation software is a very efficient and economical way to design and analyze fiber optical systems. Commercial software have been used to develop a SMF model and built a mode list. It is easily extendible and reusable according to circumstances.

Fiber Bragg gratings play a very important role in the field of communication and sensor systems. The coupled mode theory and two mode approximation are simple and accurate theories to study the grating. In this project the optical fiber Bragg gratin was modeled, simulated and discussed. The optical fiber was modeled and the field components were calculated using FIMMWAVE, and the transmission spectra was built in MatLab using CMT.

The results were presented in Chapter 6, where first the SMF and coated SMF were emerged in air and in water. From the results it can be concluded that the refractive index of a surrounding media makes a huge difference in the mode propagation response. Gold was chosen to be a metal coating, since a lot of experiments were performed using goldcoated fibers. When the gold -coated fiber was in the water, the presence of surface plasmon was observed. Then, the simulation results were compared to experimental results. 
Then the transmission spectra for various refractive indexes of surrounding media were produced for a cladding mode with effective index of 1.353209 of TFBG with a tilt angle of $6^{0}$. Then the result was compare with the experimental results done by Chan $e t$ al. [66]. The peaks in transmission spectrum are shifted with the refractive index of outside media $\left(n_{\text {eff }}\right)$ changed in both cases, and the transmission loss decreases as $n_{\text {eff }}$ was approaching the cut off value.

The last step was to create the transmission spectra for the coated TFBG. The same parameters were used except between the cladding and the surrounding media a layer of gold was placed. The results showed that the transmission shifts in a different manner compared to the uncoated TFBG. There are no experimental results that can be used to support this hypothesis.

During the project a number of problems have been encountered. The project took more time than it was expected. The computer set up took some time; the numerical model had to be modified several times until it was working properly.

Many problems remain to be solved and many careful experiments are still to be done. From the results it can be seen that the simulation can be used to analyze the problems of fiber Bragg gratings. One of the next steps would be to prove or to contradict the last hypothesis, theoretically and experimentally, built transmission spectra for different modes and different tilt angles and compare the results. Study the dependence of the tilt angle, thickness and type of coating material. All those parameters influence the outcome significantly. 


\section{References}

[1] G. A. Ball and W. W. Morey, "Continuously tunable single-mode erbium fiber laser," Optics Letters, vol. 17, pp. 420-422, 1992.

[2] F. K. Bilodeau, K. O. Hill, B. Malo, D. C. Johnson, and J. Albert, "High-returnloss narrowband all-fiber bandpass Bragg transmision filter," IEEE Photonics Technology Letters, vol. 6, pp. 80-82, 1994.

[3] K. O. Hill, B. Malo, K. A. Vineberg, F. Bilodeau, D. C. Johnson, and J. Skinner, "Efficient mode conversion in telecommunication fiber using externally written gratings," Electronics Letters, vol. 26, pp. 1270-1272, 1990.

[4] P. A. Morton, V. Mizrahi, S. G. Kosinski, L. F. Mollenauer, T. Tanbun-Ek, R. A. Logan, D. L. Coblentz, A. M. Sergent, and K. W. Wecht, "Hybrid soliton pulse source with fiber external cavity and Bragg reflector," Electronics Letters, vol. 20, pp. 561-562, 1992.

[5] E. Udd, Fiber Optic Sensors. New York: Wiley, 1991.

[6] G. E. Keiser, Optical Fiber Communications, 3 ed. New York: McGraw-Hill, 2000.

[7] G. P. Agrawal, Fiber-Optics communication systems, 3 ed. Hoboken, NJ: Wiley, 2002.

[8] V. Vali and R. W. Shorthill, "Fiber ring interferometer," Applied Optics, vol. 15, pp. 1099-1100, 1978.

[9] K. O. Hill, Y. Fujii, D. C. Johnson, and B. S. Kawaski, "Photosensitivity in optical fiber waveguides: Application to reflection filter fabrication," Applied Physics Letters, vol. 32, pp. 647-649, 15 may 19781978.

[10] G. Meltz, W. W. Morey, and W. H. Glenn, "Formation of Bragg gratings in optical fibers by a transverse holographic method," Optics Letters, vol. 14, pp. 823-825, 1989.

[11] K. O. Hill, B. Malo, F. Bilodeau, D. C. Johnson, and J. Albert, "Bragg gratings fabricated in monomode photosensitive optical fiber by UV exposure through a phase mask," Applied Physics Letters, vol. 63, p. 424, 1993.

[12] C. Chen, T. Guo, A. Laronche, and J. Albert, "Radiation mode resonances of tilted fiber Bragg gratings for high index media measurement," presented at the 19th International Conference on Optical Fibre Sensors, 2008.

[13] R. Kashyap, Fiber Bragg Gratings, 2 ed.: Elsevier Inc., 2010.

[14] A. Yariv, "Coupled-mode theory for guided-wave optics," Quantum Electronics, IEEE Journal of, vol. 9, pp. 919-933, 1973.

[15] T. Erdogan and J. E. Sipe, "Tilted fiber phase gratings," Journal of the Optical Society of America A, vol. 13, pp. 296-313, 1996.

[16] T. Erdogan, "Cladding-mode resonances in short- and long-period fiber grating filters," Journal of the Optical Society of America A, vol. 14, pp. 1760-1773, 1997.

[17] K. S. Lee and T. Erdogan, "Fiber Mode Coupling in Transmissive and Reflective Tilted Fiber Gratings," Applied Optics, vol. 39, pp. 1394-1404, 2000. 
[18] S. Lu, O. Xu, S. Feng, and S. Jian, "Analysis of radiation-mode coupling in reflective and transmissive tilted fiber Bragg gratings," Journal of the Optical Society of America A, vol. 26, pp. 91-98, 2009.

[19] A. M. Vengsarkar, J. R. Pedrazzani, J. B. Judkins, P. J. Lemaire, N. S. Bergano, and C. R. Davidson, "Long-period fiber-grating-based gain equalizers," Optics Letters, vol. 21, pp. 336-338, 1996.

[20] E. Anemogiannis, E. N. Glytsis, and T. K. Gaylord, "Transmission characteristics of long-period fiber gratings having arbitrary azimuthal/radial refractive index variations," Lightwave Technology, Journal of, vol. 21, pp. 218-227, 2003.

[21] S. A. Vasiliev and O. I. Medvedkov, "Long-period refractive index fiber gratings: properties, applications, and fabrication techniques," presented at the Advances in Fiber Optics, 2000.

[22] Y. Li, M. Froggatt, and T. Erdogan, "Volume current method for analysis of tilted fiber gratings," Lightwave Technology, Journal of, vol. 19, pp. 1580-1591, 2001.

[23] R. B. Walker, S. J. Mihailov, P. Lu, and D. Grobnic, "Shaping the radiation field of tilted fiber Bragg gratings," Journal of the Optical Society of America B, vol. 22, pp. 962-974, 2005.

[24] Y. Li and T. G. Brown, "Radiation modes and tilted fiber gratings," Journal of the Optical Society of America B, vol. 23, pp. 1544-1555, 2006.

[25] Y.-J. He, Y.-L. Lo, and J.-F. Huang, "Optical-fiber surface-plasmon-resonance sensor employing long-period fiber gratings in multiplexing," Journal of the Optical Society of America B, vol. 23, pp. 801-811, 2006.

[26] L. Yu-Chun, H. Wei-Ping, and J. Shui-Sheng, "Influence of Mode Loss on the Feasibility of Grating-Assisted Optical Fiber Surface Plasmon Resonance Refractive Index Sensors," Lightwave Technology, Journal of, vol. 27, pp. 48044808, 2009.

[27] Y.-C. Lu, R. Geng, C. Wang, F. Zhang, C. Liu, T. Ning, and S. Jian, "Polarization Effects in Tilted Fiber Bragg Grating Refractometers," Lightwave Technology, Journal of, vol. 28, pp. 1677-1684, 2010.

[28] J. U. Thomas, N. Jovanovic, R. G. Kramer, G. D. Marshall, M. J. Withford, A. Tunnermann, S. Nolte, and M. J. Steel, "Cladding mode coupling in highly localized fiber Bragg gratings II: complete vectorial analysis," Optics Express, vol. 20, pp. 21434-21449, 2012.

[29] K. O. Hill and G. Meltz, "Fiber Bragg grating technology fundamentals and overview," Journal of Lightwave Technology, vol. 15, pp. 1263-1276, 1997.

[30] R. Helan and F. Urban, "Unform fiber Bragg grating properties," http://www.feec.vutbr.cz/EEICT/2005/sbornik/03-Doktorske projekty/06Mikroelektronika_a technologie/14-helan.pdf 2006.

[31] A. Othonos and K. Kalli, Fiber Bragg Gratings. Fundamentals and Applications in telecommunications and Sensing Norwood, MA: Artech House, Inc., 1999.

[32] G. Laffont and P. Ferdinand, "Tilted short-period fibre-Bragg-grating-induced coupling to cladding modes for accurate refractometry," Measurement Science and Technology, vol. 12, pp. 765-770, 2001.

[33] T. Erdogan, "Fiber grating spectra," Journal of Lightwave Technology, vol. 15, pp. 1277-1294, 1997. 
[34] B. Malo, S. Theriault, D. C. Johnson, F. Bilodeau, J. Albert, and K. O. Hill, "Apodised in fiber Bragg grating reflectors photoimprinted using a phase mask," Electronics Letters, vol. 31, pp. 223-225, 1995.

[35] F. Ouellette, "Dispersion cancellation using linearly chirped Bragg grating filters in optical waveguides," Optics Letters, vol. 12, pp. 847-849, 1987.

[36] M. E. Fermann, K. Sugden, and I. Bennion, "High-power soliton fiber laser based on pulse width control with chirped Bragg gratings," Optics Letters, vol. 20, pp. 172-174, 1995.

[37] G. Meltz, W. W. Morey, and W. H. Glenn, "In-fiber Bragg grating tap," in Conference on Optical Fiber Communications, OFC'90, San Francisco, Calif, 1990, p. TUG1.

[38] C. Chen, L. Xiong, A. Jafari, and J. Albert, "Differential sensitivity characteristics of tilted fiber Bragg grating sensors," Proceedings of the SPIE, vol. 6004, pp. 8493, 2005.

[39] J. Albert, L.-Y. Shao, and C. Caucheteur, "Tilted Fiber Bragg Grating Sensors," Laser and Photonics Reviews, To be published, Jan 2013, DOI 10.1002/lpor.201100039.

[40] A. Cusano, D. Paladino, A. Iadicicco, S. Campopiano, and C. Caucheteur, "Single and Multiple Phase shits tilted fiber bragg gratings," Research Letters in Optics, vol. 2009, pp. 1-4, 2009.

[41] J. A. R. Williams, I. Bennion, K. Sugden, and N. J. Doran, "Fibre dispersion compensation using a chirped in-fibre Bragg grating," Electronics Letters, vol. 30, pp. 985-987, 1994.

[42] Y. Tohmori, F. Kano, H. Ishii, Y. Yoshikuni, and Y. Kondo, "Wide tuning with narrow linewidth in DFB lasers with superstructure grating (SSG)," Electronics Letters, vol. 29, pp. 1350-1351, 1993.

[43] M. Prabhu, N. S. Kim, L. Jianren, J. Xu, and K. Ueda, "Highly-efficient ultrabroadband sipercontinuum generation centered at $1484 \mathrm{~nm}$ using Raman fiber laser," presented at the Photonics West, San Jose, USA, 2001.

[44] T. Mizuochi, T. Kitayama, K. Shimizu, and K. Ito, "Interferometric crosstalk-free optical add/drop multiplexer using Mach-Zehnder-based fiber gratings," Journal of Lightwave Technology, vol. 16, pp. 265-276, 1998.

[45] H. S. Guang, "Optical phase conjugation: principles, techniques, and applications," Progress In Quantum Electronics, vol. 26, pp. 131-191, 2002.

[46] A. D. Kersey, M. A. Davis, H. J. Patrick, N. Leblanc, K. P. Koo, C. G. Askins, M. A. Putnam, and E. J. Friebele, "Fibre grating sensors," Journal of Lightwave Technology, vol. 15, pp. 1442-1463, 1997.

[47] R. Kashyap, "Photosensitive optical fibers: devices and applications," Optical Fiber Technology, vol. 1, pp. 17-34, 1994.

[48] G. A. Ball, W. W. Morey, and W. H. Glenn, "Standing-wave monomode erbium fibre laser," IEEE Photonics Technology Letters, vol. 3, pp. 613-615, 1991.

[49] J.-L. Archambault and S. G. Grubb, "Fiber Gratings in Lasers and Amplifiers," Journal of Lightwave Technology, vol. 15, pp. 1378-1389, 1997.

[50] A. Yariv, "Coupled mode theory for guided-wave optics," IEEE Journal of Quantum Electronics, vol. 9, pp. 919-933, 1973. 
[51] A. Snyder, "Coupled-mode theory for optical fibers," Journal of the Optical Society of America vol. 62, pp. 1267-1277, 1972.

[52] H. Kogelnik, "Filter response of non-uniform almost-periodic structures," Bell System Technical Journal, vol. 55, pp. 109-126, 1976.

[53] A. Yariv and P. Yeh, Photonics. Optical Electronics in Modern Communications, 6 ed. Oxford, New York: Oxford University Press, Inc., 2007.

[54] C.-L. Chen, Foundations for Guided-Wave Optics. Hoboken, New Jersey: John Wiley \& Sons, Inc., 2007.

[55] R. Zengerle and O. Leminger, "Phase-Shifted Bragg-Grating Filters with Improved Transmission Characteristics," Journal of Lightwave Technology, vol. 13, pp. 2354-2358, 1995.

[56] C. Y. H. Tsao, D. N. Payne, and W. A. Gambling, "Modal characteristics of threelayered optical fiber waveguides: a modified approach," Journal of the Optical Society of America A, vol. 6, pp. 555-563, 1989.

[57] A. W. Snyder and W. R. Young, "Modes of optical waveguides," Journal of the Optical Society of America, vol. 68, pp. 297-309, 1978.

[58] J. U. Thomas, "Mode control with ultra-short pulse written fiber Bragg gratings," doctor rerum naturalium, Physikalisch-Astronomischen Facultat, FriedrichSchiller-Universitat Jena, Jena, Germany, 2012.

[59] J. Thomas, N. Jovanovic, R. G. Becker, G. D. Marshall, M. J. Withford, A. Tunnermann, S. Nolte, and M. J. Steel, "Cladding mode coupling in highly localized fiber Bragg gratings: modal properties and transmission spectra," Optics Express, vol. 19, pp. 325-341, 2011.

[60] Photon Design, "Fimmwave v5.2.3," user manual.

[61] A. S. Sudbo, "Film mode matching: a versatile numerical method for vector mode field calculations in dielectric waveguides "Pure and Applied Optics: Journal of the European Optical Society Part A, vol. 2, pp. 211-233, 1993.

[62] D. M. Etter, Engineering problem solving with MATLAB: Prentice Hall, 1993.

[63] T.-C. Poon and T. Kim, Engineering Optics with MATLAB. Singapore: World Scientific Publishing Co. Pte. Ltd, 2006.

[64] K. Welford, "A method of Attanuated Reflection," in Surface Plasmon Polaritons, 9 ed UK: Techno House, 1988.

[65] Y. Shevchenko, C. Chen, M. A. Dakka, and J. Albert, "Polarization-selective grating excitation of plasmons in cylindrical optical fibers," Optics Letters, vol. 35, pp. 637-639, 2010.

[66] C.-F. Chan, C. Chen, A. Jafari, A. Laronche, D. J. Thomson, and J. Albert, "Optical fiber refractometer using narrowband cladding-mode resonance shifts," Applied Optics, vol. 46, pp. 1142-1149, 2007. 


\section{Appendices}

\section{Appendix A}

\section{A.1 User Manual}

1. Install FIMMWAVE v 5.3.8

2. Install MatLab

3. Open SMF28_50nmAu.prj for gold-coated fiber or SMF28.prj for bare fiber 4. Add the materials database to the FIMMWAVE. To do so add refbase.m to the MATLAB folder, open FIMMWAVE, click on Solver, Add database and choose refbase.m

5. Click on Edit, Waveguide or on button and edit the properties of the waveguide. As it is described in the Chapter 2.2 (pg. 26) of the Fimmwave user manual. Click on each layer and edit its properties in the lower portion of the window using Table 6.1 for bare fiber. Note to click on the mat instead of rix(iso) option for each layer. When dealing with gold-coated fiber, use refractive index for gold $0.55-i 11.5$. And click OK.

6. Now go on to lactation of the modes of the waveguide. Use user manual Chapter 2.2.3 and in the FWG waveguide editor click button to open MOLAB options. Choose FDM complex solver. Define the range for the effective indexes of the modes and the amount of modes you wish to calculate. Note it takes longer to calculate large amount of modes. For the mode profile resolution put $n x=20$ and $n y=20$. Click Edit solver parms. Edit the wavelength (Lambda (um)), m-order and p-order and resolution if needed. Click OK. And in the MOLAB options click OK. Now click button and 
click on "Build list" button and click "start". Note: Click "yes" if you wish to delete all existing modes and you want to start a new list or click "no" if you wish to add more modes to the existing list.

7. After the mode list has been generated choose the mode you wish to examine and click on "Detail". If the m-order and p-order are the numbers you are looking for, then click OK. Then click on "Inspect". In "plot area" region pill in the fields for xmin=70 $x \max =90$ ymin $=70$ ymax $=90$ and $n x$ ny $=100$ and 100 . In "AMF file" region add format string $=\% 10.15 \mathrm{f}$ (the number of decimal places after 10 . determines the amount of decimal places each value of a matrix will have). Click on "Write AMF". Save this file in your MATLAB folder. Repeat this procedure for core mode and for cladding mode with different effective indexes and different $m$-order values as described in Chapter 6.2.1.

8. Open FW_complex.m file in MatLab. Make sure that $\mathrm{s} 0=$ is the core mode AMF file and sm is the cladding modes AMF files. In this file it is possible to change the tilt angle on line 14 .

Note: change the number of the file 'FileName_\#.txt' in line 181 each time you run the simulation (for each refractive index of surrounding media change). Also each time you change the refractive index of surrounding media, save (copy/paste) the result values from the Command window into an Excel file and record the value for the surrounding media refractive index. In Excel file it is possible to monitor all the result values and plot all necessary graphs. After all the desired calculations were done for all he refractive index changes of the outside media, the transmission spectra can be built as a function of wavelength.

9. Open plot_matrixes.m file in Matlab and run it. 
Note: make sure that the $\mathrm{x}$ values in line 5 are the same as the values in line 158 of FW_complex.m file.

\section{A.2 MatLab Codes}

FW_complex.m code:

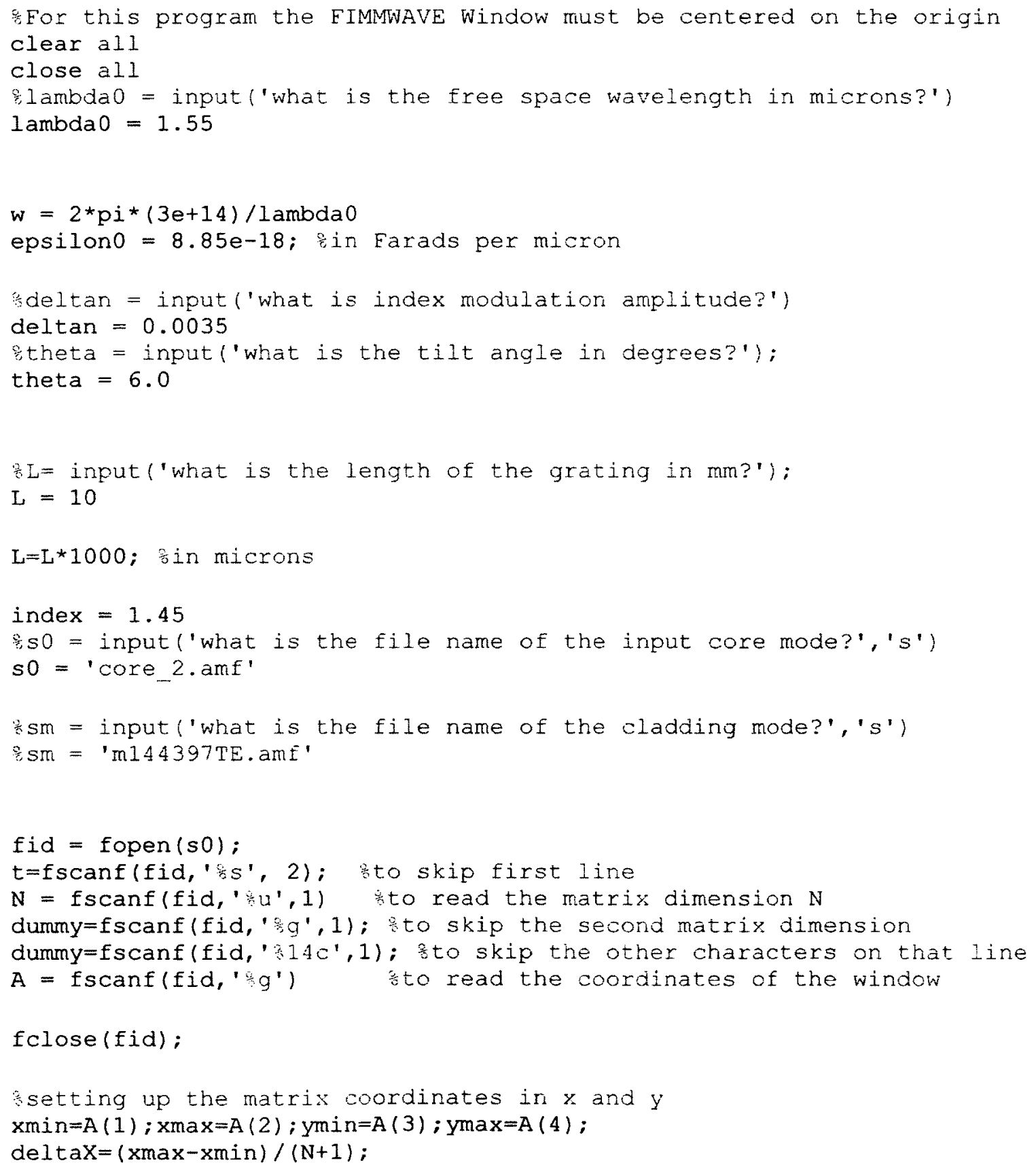




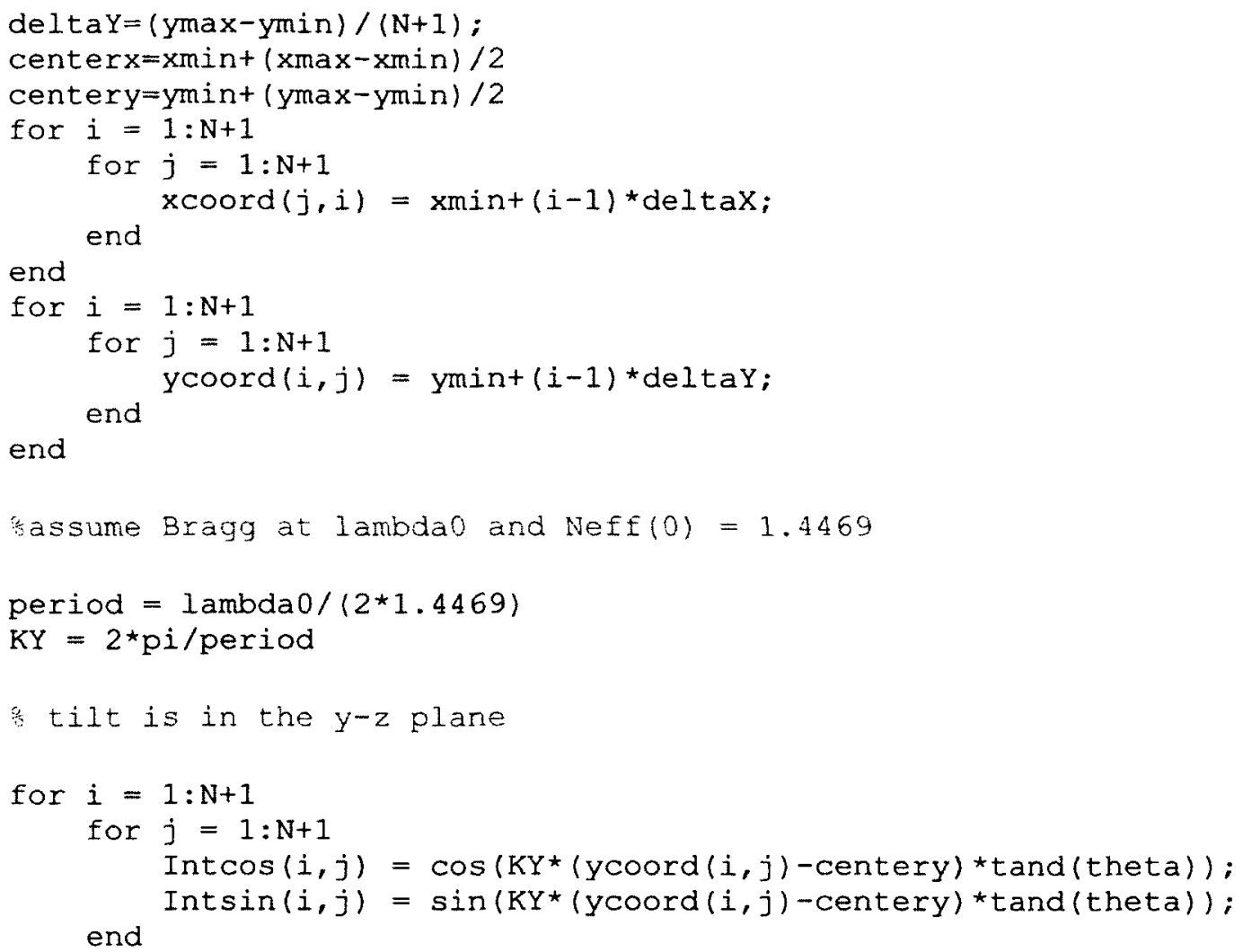




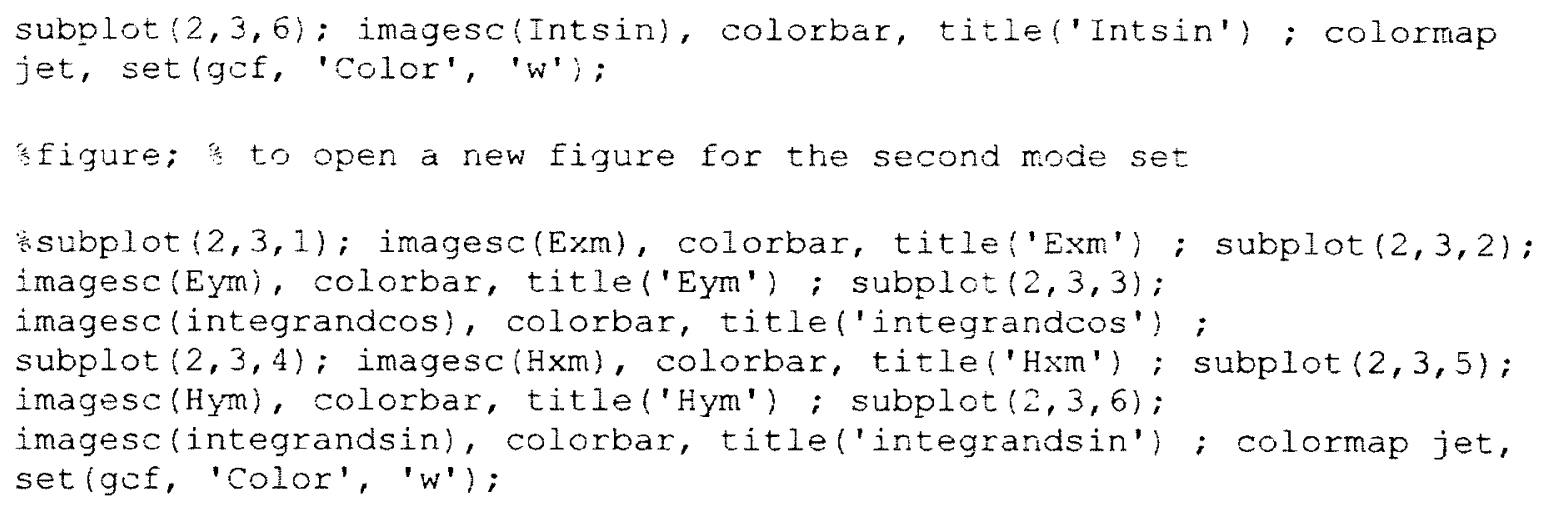




\section{plot_matrixes.m Code:}

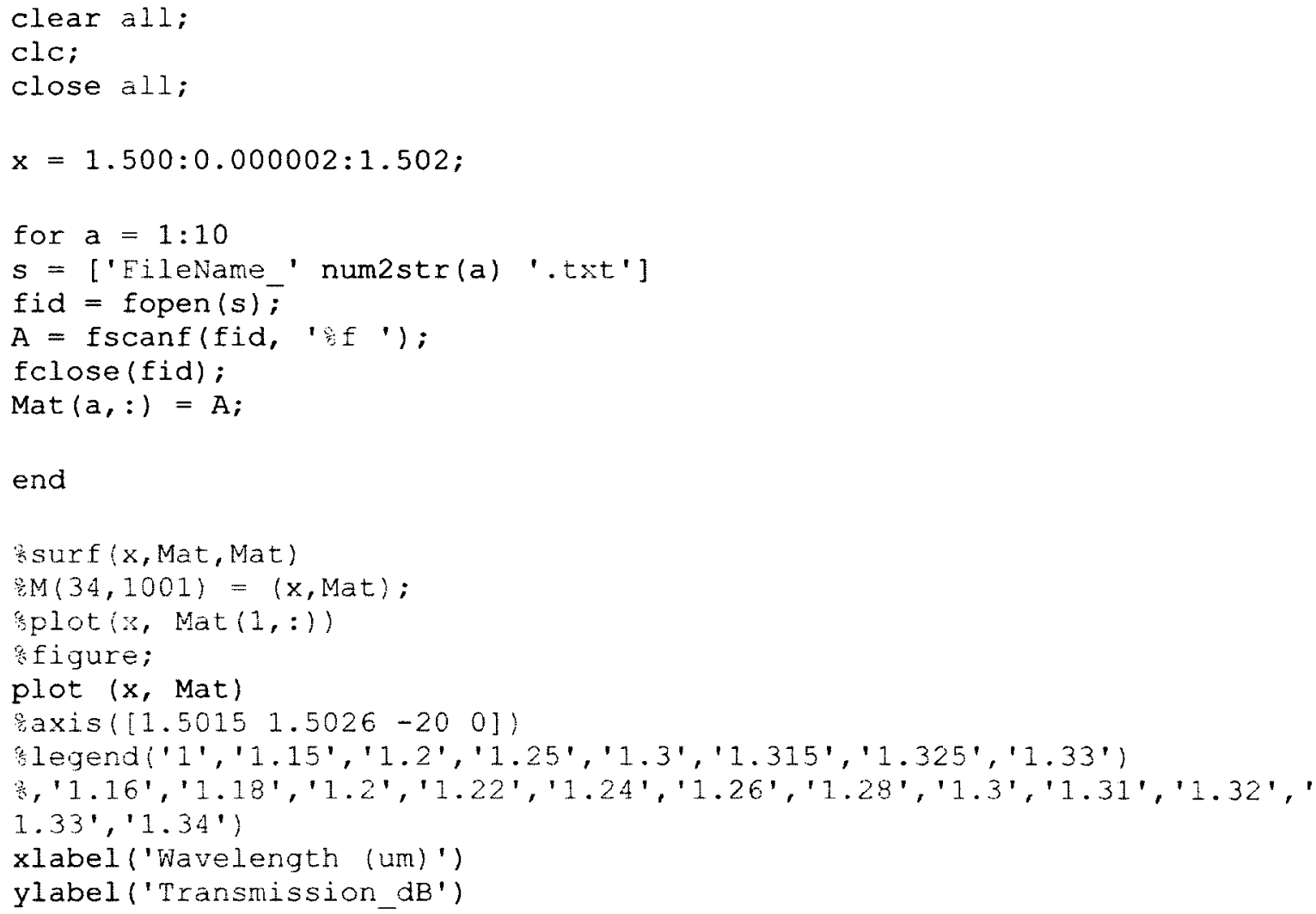

